\title{
ALMOST PERIODIC AND STRONGLY STABLE SEMIGROUPS OF OPERATORS
}

\author{
VŨ QUÔC PHÓNG \\ Department of Mathematics, Ohio University \\ Athens, Ohio 45701, U.S.A. \\ E-mail: qvu@oucsace.cs.ohiou.edu
}

1. Introduction. This paper is chiefly a survey of results obtained in recent years on the asymptotic behaviour of semigroups of bounded linear operators on a Banach space. From our general point of view, discrete families of operators $\left\{T^{n}: n=0,1, \ldots\right\}$ on a Banach space $X$ (discrete one-parameter semigroups), one-parameter $C_{0}$-semigroups $\{T(t): t \geq 0\}$ on $X$ (strongly continuous one-parameter semigroups), are particular cases of representations of topological abelian semigroups. Namely, given a topological abelian semigroup $S$, a family of bounded linear operators $\{T(s): s \in S\}$ is called a representation of $S$ in $B(X)$ if: (i) $T(s+t)=T(s) T(t)$; (ii) For every $x \in X, s \mapsto T(s) x$ is a continuous mapping from $S$ to $X$.

The central result which will be discussed in this article is a spectral criterion for almost periodicity of semigroups, obtained by Lyubich and the author [40] for uniformly continuous representations of arbitrary topological abelian semigroups (thus including the case of single bounded operators and several commuting bounded operators), and for $C_{0}$-semigroups [41], and by Batty and the author [9] for arbitrary strongly continuous representations of suitable locally compact abelian semigroups. An immediate consequence of this result is a Stability Theorem, obtained, for single operators and $C_{0}$-semigroups, also by Arendt and Batty [1] independently. The proof in [1] uses a Tauberian theorem for the Laplace-Stieltjes transforms and transfinite induction. Methods of this type can also be used to prove the almost periodicity result for $C_{0^{-}}$ semigroups [8], but seem not suitable for commuting semigroups, and will not be discussed in this article.

We also refer the reader to a recent survey article of Batty [6], where some developments are described which are not included here.

1991 Mathematics Subject Classification: 47D03, 47D07, 34C27, 43A65.

The paper is in final form and no version of it will be published elsewhere. 
2. The semigroup $\left\{T^{n}: n \in \mathbb{Z}_{+}\right\}$. In this section, $T$ is a bounded linear operator on a (complex) Banach space $X, T \in B(X)$. The spectrum, point spectrum, and resolvent set of the operator $T$ are denoted by $\sigma(T), P \sigma(T)$ and $\varrho(T)$, respectively. If $\lambda \in P \sigma(T)$, we put $X_{\lambda}=\{x \in X: T x=\lambda x\}$. The operator $T$ is called power-bounded if the discrete semigroup $\left\{T^{n}: n \in \mathbb{Z}_{+}\right\}$is bounded, i.e. $\sup _{n \in \mathbb{Z}_{+}}\left\|T^{n}\right\|<\infty$, and $T$ is called strongly stable if $\left\|T^{n} x\right\| \rightarrow 0$ as $n \rightarrow \infty, \forall x \in X$. For a power-bounded operator $T$ we denote by $E \sigma(T)$ the ergodic spectrum of $T$, i.e. $E \sigma(T)$ is a subset of the unit circle, $\mathbb{T}$, defined by

$$
E \sigma(T)=\left\{\lambda \in \mathbb{T}: \exists x^{*} \in X^{*}: x^{*} \neq 0, T^{*} x^{*}=\lambda x^{*}, x^{*} \mid X_{\lambda} \equiv 0\right\} .
$$

1. Limit isometric semigroup. A central role in our methods of proofs is played by the following construction, valid for power-bounded operators on Banach spaces. This construction was used by Sklyar and Shirman [39] who obtained the result contained in Corollary 3.8 for uniformly continuous semigroups, and by Lyubich and the author [31], [40-41]. Similar construction for operators of the so-called class $C_{1}$ (i.e. when $\left\|T^{n} x\right\| \not \rightarrow 0$, $\forall x \in X)$ was used by Beauzamy [10] and, in the case $X$ is a Hilbert space, by Kérchy [25], in connection with the problems of invariant subspaces. In this form, it is contained in $[42]$.

Proposition 2.1. Let $T$ be a power-bounded operator on $X$. There exist another Banach space $E$, a continuous linear operator $Q: X \rightarrow E$, with dense range, and an isometric operator $V$ on $E$ such that:

(i) $Q T=V Q$;

(ii) $\sigma(V) \subset \sigma(T), P \sigma(T) \cap \mathbb{T} \subset P \sigma(V), P \sigma\left(V^{*}\right) \subset P \sigma\left(T^{*}\right)$;

(iii) $\|Q x\|_{E}=\lim \sup _{n \rightarrow \infty}\left\|T^{n} x\right\|$.

Moreover, such $E$ and $V$ are unique in the sense that if $E^{\prime}$ is another Banach space and $V^{\prime}: E^{\prime} \rightarrow E^{\prime}$ is an isometric operator satisfying (i)-(iii), then there is an invertible isometric operator $U: E \rightarrow E^{\prime}$ such that $U V U^{-1}=V^{\prime}$.

Proof. Introducing an equivalent norm $\|x\|=\sup _{n \geq 0}\left\|T^{n} x\right\|$ in $X$, we can assume that $T$ is a contraction. Let $l(x)$ be a semi-norm on $X$ defined by $l(x) \equiv \lim _{n \rightarrow \infty}\left\|T^{n} x\right\|$, $x \in X, \widehat{X}=X / \operatorname{ker} l$. Further, let $Q$ denote the natural homomorphism from $X$ to $\widehat{X}$ and $\widehat{T}: \widehat{X} \rightarrow \widehat{X}$ be defined by $\widehat{T}(Q x)=Q(T x)$. The semi-norm $l$ induces in the quotient space $\widehat{X}$ a norm $\widehat{l}$ in a natural way, i.e. $\widehat{l}(Q x) \equiv l(x)$. Clearly, $\widehat{T}$ is an isometry on the normed space $(\widehat{X}, \widehat{l})$. Let $E$ be the completion of $\widehat{X}$ and let $V$ be the extension of $\widehat{T}$ to the whole $E$ by continuity. Then $V$ is isometric, $Q$ is a bounded linear operator from $X$ to $E$ with dense range and (i) holds. The property (iii) holds by construction. To show that (ii) holds, let $A$ be a bounded linear operator on $X$ which commutes with $T$. Then $A$ induces an operator $\widehat{A}$ on $E$ by $\widehat{A}(Q x)=Q(A x)$, which is bounded, since $\|A\|=\sup \{\widehat{l}(A x): l(x)=1\} \leq \sup \{\|A\| l(x): l(x)=1\}=\|A\|$. Hence, if $\lambda \in \varrho(T)$, and $A=(\lambda-T)^{-1}$, then $\lambda \in \varrho(\widehat{T})$ and $\widehat{A}=(\lambda-\widehat{T})^{-1}$. Thus, $\sigma(U) \subset \sigma(T)$. If $T x=\lambda x$, $|\lambda|=1$, then $V(Q x)=\lambda Q x, Q x \neq 0$, hence $\lambda \in P \sigma(V)$. If $V^{*} \phi=\mu \phi, \phi \neq 0$, then the functional $\varphi \in X^{*}$ defined by $\varphi(x) \equiv \phi(Q x)$ satisfies $T^{*} \varphi=\lambda \varphi, \varphi \neq 0$. Thus, $\operatorname{P\sigma }\left(V^{*}\right) \subset P \sigma\left(T^{*}\right)$. 
Finally, if $E^{\prime}$ is another Banach space, $Q^{\prime}: X \rightarrow E^{\prime}$ is a corresponding map, and $V^{\prime}: E^{\prime} \rightarrow E^{\prime}$ is an isometry satisfying (i)-(iii), then the operator $U: E \rightarrow E^{\prime}$ defined by $U(Q x)=Q^{\prime} x$ is an isometry (by (iii)) with dense domain and dense range. Therefore, $U$ is an invertible isometry, and $U V(Q x)=U(Q T x)=Q^{\prime} T x=V^{\prime} Q^{\prime} x=V^{\prime} U(Q x)$, so that $U V=V^{\prime} U$.

Proposition 2.1 gives the following characterization of strong stability of the discrete semigroup $\left\{T^{n}: n \in \mathbb{Z}_{+}\right\}$obtained in [44, Theor. 5.1]. $\left(^{1}\right)$

ThEOREM 2.2. Let $T$ be a power-bounded operator on $X$. Then the following are equivalent:

(1) $T$ is strongly stable;

(2) There do not exist $\left\{x_{n}^{*}\right\}_{n=-\infty}^{0} \subset X^{*}, x_{0}^{*} \neq 0$ such that $\sup _{n \leq 0}\left\|x_{n}^{*}\right\|<\infty$ and $\left(T^{*}\right)^{n} x_{-n}^{*}=x_{0}^{*}, \forall n \geq 0$

(3) There do not exist $\left\{x_{n}^{*}\right\}_{n=-\infty}^{\infty} \subset X^{*}, x_{0}^{*} \neq 0$, such that

$$
\sup _{n \in \mathbb{Z}}\left\|x_{n}^{*}\right\|<\infty \quad \text { and } \quad\left(T^{*}\right)^{k} x_{n}^{*}=x_{k+n}^{*}, \quad \forall n \in \mathbb{Z}, k \geq 0 ;
$$

Proof. $(1) \Rightarrow(2)$. Let $T$ be strongly stable and assume, on the contrary, that $x_{-n}^{*}$, $n=0,1, \ldots$, is a bounded sequence in $X^{*}$ such that $\left(T^{*}\right)^{n} x_{-n}^{*}=x_{0}^{*} \neq 0$. Then we have, for every $x \in X, 0=\lim _{n \rightarrow \infty}\left|\left\langle T^{n} x, x_{-n}^{*}\right\rangle\right|=\left|\left\langle x,\left(T^{*}\right)^{n} x_{-n}^{*}\right\rangle\right|=\left|\left\langle x, x_{0}^{*}\right\rangle\right|$, which is a contradiction.

$(2) \Rightarrow(3)$ is trivial.

$(3) \Rightarrow(1)$. Assume that $T$ is not strongly stable. Then the limit isometric operator $V$ constructed in Proposition 2.1 is nontrivial (i.e. $E \neq 0$ ). Let $z_{0}^{*}$ be any nonzero element in $E^{*}$. Define inductively, for each $n=1,2, \ldots$, a functional $z_{-n}^{*}$ on $V(E)$ by

$$
\left\langle V z, z_{-n}^{*}\right\rangle=\left\langle z, z_{-n+1}^{*}\right\rangle \text {. }
$$

Then $\left|\left\langle V z, z_{-n}^{*}\right\rangle\right| \leq\left\|z_{-n+1}^{*}\right\|_{E^{*}}\|z\|_{E}=\left\|z_{-n+1}^{*}\right\|_{E^{*}}\|V z\|_{E}$, hence $z_{-n}^{*}$ is uniformly bounded by $\left\|z_{0}^{*}\right\|$. By the Hahn-Banach Theorem, there exist norm-preserving extensions of $z_{-n}^{*}$ to the whole $E$, which we denote by the same $z_{-n}^{*}$. Clearly, $\left(V^{*}\right)^{k} z_{-n}^{*}=$ $z_{-n+k}^{*}, \forall k, n \geq 0$. Let $x_{-n}^{*}, n=0,1, \ldots$ be corresponding elements of $X^{*}$ defined by $\left\langle x, x_{-n}^{*}\right\rangle=\left\langle Q x, z_{-n}^{*}\right\rangle$, and let $x_{n}^{*}=\left(T^{*}\right)^{n} x_{0}^{*}, \forall n>0$. Then, as can be verified directly, $\left\{x_{n}^{*}\right\}$ is uniformly bounded in $X^{*}$ and satisfies condition (2.1).

2. Almost periodicity and stability. Recall that a discrete semigroup $\left\{T^{n}: n \in Z_{+}\right\}$, generated by a single operator $T$, is called almost periodic if the orbit $\left\{T^{n} x: n \in \mathbb{Z}_{+}\right\}$is relatively compact, $\forall x \in X$. If $T$ is invertible, then the group $\left\{T^{n}: n \in \mathbb{Z}\right\}$, generated by $T$, is called an almost periodic group if $\left\{T^{n} x: n \in \mathbb{Z}\right\}$ is relatively compact, $\forall x \in X$. If $\left\{T^{n}: n \in \mathbb{Z}\right\}$ is an almost periodic group, then $X=\overline{\operatorname{span}}\{x \in X: \exists \lambda \in \mathbb{T}: T x=\lambda x\}$. If $T$ is strongly stable, then $\left\{T^{n}: n \in \mathbb{Z}_{+}\right\}$is also almost periodic. The following theorem, usually known as the de Leeuw-Glicksberg Decomposition, gives a description of the structure of almost periodic discrete semigroups (see [26], [30]).

$\left({ }^{1}\right)$ This result was proved in [44] under some additional condition (i) or (ii). That this additional condition is not necessary has also been observed independently (by a different argument) by C. J. K. Batty, Z. Brzeźniak and D. Greenfield [7]. 
THEOREM 2.3. If a discrete semigroup $\left\{T^{n}: n \in \mathbb{Z}_{+}\right\}$on $X$ is almost periodic, then $X$ has the following decomposition into a direct topological sum:

$$
X=X_{\mathrm{b}}+X_{\mathrm{s}},
$$

where $X_{\mathrm{s}}=\left\{x \in X: \lim _{n \rightarrow \infty}\left\|T^{n} x\right\|=0\right\}$, and $X_{\mathrm{b}}=\overline{\operatorname{span}}\{x \in X: \exists \lambda \in \mathbb{T}: T x=\lambda x\}$. Moreover, $T \mid X_{\mathrm{b}}$ is invertible and $\left\{\left(T \mid X_{\mathrm{b}}\right)^{n}: n \in \mathbb{Z}\right\}$ is a bounded almost periodic group. The projection $P: X \rightarrow X_{\mathrm{b}}$ such that $\operatorname{ker} P=X_{\mathrm{s}}$ is in the closure of $\left\{T^{n}: n \in \mathbb{Z}_{+}\right\}$ in the strong operator topology. If , in addition, $T$ is a contraction, then $T \mid X_{\mathrm{b}}$ is an invertible isometry and $\|P\|=1$ (if $P \neq 0)$.

The subspaces $X_{\mathrm{b}}$ and $X_{\mathrm{s}}$ are called boundary and stable subspaces of $T$, respectively, and the projection $P$ is called the boundary projection [30]. In general, if $\left\{T^{n}: n \in \mathbb{Z}_{+}\right\}$ is bounded, but not almost periodic, then we still can define the subspaces $X_{\mathrm{s}}$ and $X_{\mathrm{b}}$ as in Theorem 2.3, and let $X_{\mathrm{ap}}=\overline{\operatorname{span}}\left\{y+z: y \in X_{\mathrm{b}}, z \in X_{\mathrm{s}}\right\}$. Then, as is easily seen, $\left\{T^{n} \mid X_{\text {ap }}: n \in \mathbb{Z}_{+}\right\}$is an almost periodic semigroup. Therefore, applying Theorem 2.3 to this semigroup we see that in fact $X_{\mathrm{ap}}=X_{\mathrm{b}} \dot{+} X_{\mathrm{s}}$.

If, in the construction in the proof of Proposition 2.1, we take, instead of $X /$ ker $l$, the quotient space $X / X_{\text {ap }}$, with an appropriate norm, then we obtain the following proposition.

Proposition 2.4. Let $T$ be a power-bounded operator on $X$. There exist another Banach space $F$, a continuous linear operator $R: X \rightarrow F$, with dense range, and an isometric operator $U$ on $F$ such that:

(i) $R T=U R$;

(ii) $\sigma(U) \subset \sigma(T), P \sigma(U)=\emptyset, P \sigma\left(U^{*}\right) \subset P \sigma\left(T^{*}\right), P \sigma\left(U^{*}\right) \cap \mathbb{T} \subset E \sigma(T)$;

(iii) $\|R x\|_{E}=\lim \sup _{n \rightarrow \infty} \inf _{y \in X_{\mathrm{b}}}\left\|T^{n}(x-y)\right\|$.

Moreover, such $F$ and $U$ are unique in the sense that if $F^{\prime}$ is another Banach space and $U^{\prime}: F^{\prime} \rightarrow F^{\prime}$ is an isometric operator satisfying (i)-(iii), then there is an invertible isometric operator $W: F \rightarrow F^{\prime}$ such that $W U W^{-1}=U^{\prime}$.

The operator $U$ can be called the limit isometric operator of $T$ with continuous spectrum.

Pro of. The proof of Proposition 2.4 is an adaptation of the proof of Proposition 2.1. The modifications are that now we take the quotient space $\widehat{X}=X / X_{\text {ap }}$. Then $T$ induces an operator $\widehat{T}$ in $\widehat{X}$, which is an isometry in the norm in $\widehat{X}$ defined by

$$
\|\widehat{x}\| \mid=\limsup _{n \rightarrow \infty} \inf _{y \in X_{\mathrm{ap}}}\left\|T^{n} x-y\right\| .
$$

Let $F$ be the completion of $\widehat{X}$ with respect to the introduced norm, and let $U$ be the extension of $\widehat{T}$ from $\widehat{X}$ to $F$ by continuity. Then the properties (i)-(iii) can be verified as in the proof of Proposition 2.1.

The following theorem, which gives a spectral condition for almost periodicity of a discrete semigroup $\left\{T^{n}: n \in \mathbb{Z}_{+}\right\}$, can be proved by applying Proposition 2.4 and the theory of discrete groups of isometric operators. One earlier result of this theory is the 
Gelfand Theorem, that if $V$ is an isometry on $X$, and $\sigma(V)$ is a single point $\lambda$, then $V=\lambda I$ (see [20], [23], [50]).

THEOREM 2.5 ([40]). If $T$ is a power-bounded operator on a Banach space $X$ such that $\sigma(T) \cap \mathbb{T}$ is countable and $E \sigma(T) \cap \mathbb{T}$ is empty, then the discrete semigroup $\left\{T^{n}: n \in \mathbb{Z}_{+}\right\}$ is almost periodic.

Proof. Assuming the contrary, we can construct the Banach space $F \neq\{0\}$ and the operator $U$ as in Proposition 2.4. Then $U$, and hence $U^{*}$, is an isometric operator with a countable spectrum. If $\lambda$ is an isolated point of $\sigma(U)$, then there is $\phi \in F^{*}, \phi \neq 0$, such that $U^{*} \phi=\lambda \phi$. Let $\varphi \in X^{*}$ be defined as $\langle x, \varphi\rangle \equiv\langle R x, \phi\rangle$. Then, as can easily be seen, $T^{*} \varphi=\lambda \varphi, \varphi \mid X_{\lambda}=0$, so $\lambda \in E \sigma(T)$, which is a contradiction.

Remark 2.6. It is well known that, for a power-bounded operator $T$ and $\lambda \in \mathbb{T}$, $\lambda \notin E \sigma(T)$ if and only if the semigroup $\left\{\lambda^{-n} T^{n}: n \in \mathbb{Z}_{+}\right\}$is ergodic, i.e. $\frac{1}{n} \sum_{k=0}^{n-1} \lambda^{-k} T^{k}$ converges to the projection $P_{\lambda}$ (on the subspace $X_{\lambda}=\{x: T x=\lambda x\}$ ) (see e.g. [26], [30]). In particular, $\frac{1}{n} \sum_{k=i}^{n+i-1} \lambda^{-k} T^{k} x$ converges to $P_{\lambda} x$, uniformly in $i$. Conversely, if the means $\frac{1}{n} \sum_{k=i}^{n+i-1} \lambda^{-k} T^{k} x$ converge for some $i \in \mathbb{Z}_{+}$, then they converge uniformly in $i$, and, therefore, the restriction of $\left\{\lambda^{-n} T^{n}: n \in \mathbb{Z}_{+}\right\}$to $\overline{\operatorname{span}}\left\{T^{n} x: n \in \mathbb{Z}_{+}\right\}$is ergodic. Therefore, the condition $E \sigma(T) \cap \mathbb{T}=\emptyset$ in Theorem 2.5 can be replaced by the following equivalent condition: for each $\lambda \in \sigma(T) \cap \mathbb{T}$, the semigroup $\left\{\lambda^{-k} T^{k}: k \in \mathbb{Z}_{+}\right\}$is ergodic.

Theorem 2.5 implies the following corollary, which can also be proved directly by applying the same argument and Proposition 2.1. This result was obtained in [40], [41], and independently in [1].

Corollary 2.7. If $T$ is a power-bounded operator on a Banach space $X$ such that $\sigma(T) \cap \mathbb{T}$ is countable and $P \sigma\left(T^{*}\right) \cap \mathbb{T}$ is empty, then $T$ is strongly stable, i.e. $\left\|T^{n} x\right\| \rightarrow 0$ as $n \rightarrow \infty, \forall x \in X$.

Remark 2.8. It is a well known theorem of Sz.-Nagy and Foiaş [34] that if $T$ is a completely non-unitary (c.n.u.) contraction on a Hilbert space and if $m(\sigma(T) \cap \mathbb{T})=0$, where $m$ is the Lebesgue measure on $\mathbb{T}$, then $T$ is strongly stable. However, this result does not apply to power-bounded operators, since power-bounded operators are not, in general, similar to contractions [19], [21].

Let $W(\mathbb{T})$ be the Wiener algebra, i.e. $W(\mathbb{T})=\left\{f(z)=\sum_{n=-\infty}^{\infty} a_{n} z^{n}:\|f\|_{W} \equiv\right.$ $\left.\sum_{n=-\infty}^{\infty}\left|a_{n}\right|<\infty\right\}$. A function $f \in W(\mathbb{T})$ is called a function of spectral synthesis w.r.t. a closed subset $\Delta$ of $\mathbb{T}$ if there is a sequence $g_{n} \in W(\mathbb{T})$ such that $\left\|g_{n}-f\right\|_{W} \rightarrow 0$ as $n \rightarrow \infty$ and $g_{n}$ vanishes on a neighborhood $\mathcal{U}_{n}$ of $\Delta$, for each $n$. The following theorem, initially obtained by Katznelson and Tzafriri [24] as a consequence of a Tauberian theorem for power series, can also be proved by applying Proposition 2.1 [42].

THEOREM 2.9. If $T$ is a power-bounded operator and if $f(z)=\sum_{n=0}^{\infty} a_{n} z^{n} \in W(\mathbb{T})$ is a function of spectral synthesis with respect to $\sigma(T) \cap \mathbb{T}$, then $\left\|T^{n} f(T)\right\| \rightarrow 0$ as $n \rightarrow 0$.

Proof. First we make an observation that Theorem 2.9 follows from the following, formally weaker, assertion: under the conditions of Theorem 2.9,

$$
\left\|T^{n} f(T) x\right\| \rightarrow 0 \quad \text { as } n \rightarrow \infty, \forall x \in X .
$$


In fact, if $(*)$ is proved, then, in order to get the desired conclusion that $\left\|T^{n} f(T)\right\| \rightarrow 0$ as $n \rightarrow \infty$, it remains to apply $(*)$ to the operator $\widetilde{T}: B(X) \rightarrow B(X)$, defined by $\widetilde{T} Y=T Y$ for all $Y \in B(X)$. Now, to show that $\left\|T^{n} f(T) x\right\| \rightarrow 0$, one can assume that $\left\|T^{n} x\right\| \not \rightarrow 0$, and use the construction of Proposition 2.1. Since $\sigma(V) \subset \sigma(T)$, it follows that $f(V)=0$, that is, $\left\|T^{n} f(T) x\right\| \rightarrow 0$ as $n \rightarrow \infty, \forall x \in X$.

Theorem 2.9 gives the following corollary.

Corollary 2.10. If $T$ is power-bounded and $\sigma(T) \cap \mathbb{T} \subset\{1\}$, then $\left\|T^{n+1}-T^{n}\right\| \rightarrow 0$ as $n \rightarrow \infty$.

3. Behaviour of individual elements. It is sometime possible to make statements about almost periodicity and stability of an individual element $x$ in $X$, i.e. of a sequence $\left\{x_{n} \equiv\right.$ $\left.T^{n} x: n \in \mathbb{Z}_{+}\right\}$.

First, we observe that from Theorem 2.5 and Remark 2.6 the following statement immediately follows.

Proposition 2.11 ([15]). If $T$ is power-bounded, $\sigma(T) \cap \mathbb{T}$ is countable, and if $x$ is a vector in $X$ such that the means

$$
\frac{1}{n} \sum_{k=0}^{n-1} \lambda^{-k} T^{k} x
$$

converge for all $\lambda \in \sigma(T) \cap \mathbb{T}$, then $\left\{T^{n} x: n \in \mathbb{Z}_{+}\right\}$is an asymptotically a.p. sequence. In particular, if the above means converge to 0 , for all $\lambda \in \sigma(T) \cap \mathbb{T}$, then $\left\|T^{n} x\right\| \rightarrow 0$ as $n \rightarrow \infty$.

Remark 2.12. At this point, the reader may observe some discrepancy in the terminology. If $T$ is invertible and $\left\{T^{n}: n \in \mathbb{Z}\right\}$ is an almost periodic group, then the sequence $\left\{x_{n}=T^{n} x: n \in \mathbb{Z}\right\}$ is almost periodic, i.e. the family $\left\{x_{n+k}: n \in \mathbb{Z}\right\}_{k \in \mathbb{Z}}$ is relatively compact in $l^{\infty}(\mathbb{Z}, X)$, the Banach space of bounded $X$-valued sequences. If the semigroup $\left\{T^{n}: n \in \mathbb{Z}_{+}\right\}$is almost periodic, then the sequence $\left\{x_{n} \equiv T^{n} x: n \in \mathbb{Z}_{+}\right\}$is asymptotically almost periodic (i.e., there is an almost periodic sequence $\left\{y_{n}: n \in \mathbb{Z}\right\}$ such that $\left\|x_{n}-y_{n}\right\| \rightarrow 0$ as $\left.n \rightarrow \infty\right)$, but in general it is not almost periodic, i.e. it may not be extendable to an almost periodic sequence $\left\{x_{n}: n \in \mathbb{Z}\right\}$. Therefore, a semigroup $\left\{T^{n}: n \in \mathbb{Z}_{+}\right\}$which is almost periodic in our definition would be more exactly called asymptotically almost periodic. We preferred to follow the established terminology because it is commonly accepted in the literature.

From Proposition 2.11, and by introducing a suitable norm in $L \equiv \operatorname{span}\{x$ : $\left.\sup _{n \geq 0}\left\|T^{n} x\right\|<\infty\right\}$, we can prove the following individual variants of Theorems 2.5, 2.9 , in which $T$ is not assumed to be power-bounded, or even to be bounded.

TheOREM 2.13 ([15]). Let $T$ be an arbitrary operator on $X$ such that $\sigma(T) \cap \mathbb{T}$ is countable. If $x \in \bigcap\left\{D\left(T^{n}\right): n \geq 0\right\}$ is such that the sequence $\left\{T^{n} x: n \in \mathbb{Z}_{+}\right\}$is bounded, and if for every $\lambda \in \sigma(T) \cap \mathbb{T}$ the means

$$
\frac{1}{n} \sum_{k=j}^{n-1+j} \lambda^{k} T^{k} x
$$


converge uniformly in $j \geq 0$, then $\left\{T^{n} x: n \in \mathbb{Z}_{+}\right\}$is an asymptotically almost periodic sequence. Moreover, if the above means converge to 0 for all $\lambda \in \sigma(T) \cap \mathbb{T}$, then $\left\|T^{n} x\right\| \rightarrow$ 0 as $n \rightarrow \infty$.

Proof. Let $\|y\| \|$ be a new norm in $L$ defined by

$$
\|y\|=\sup _{n \geq 0}\left\|T^{n} x\right\|,
$$

and let $\widetilde{L}$ be the completion of $L$ in $\|\cdot \mid\|$. It is easy to see that $\|T y\| \leq\|y\|, \forall y \in L$, i.e. $T$ is a contraction in $L$, which therefore can be extended by continuity to a contraction, denoted by $\widetilde{T}$, on $L$. If $A: X \rightarrow X$ is a bounded operator commuting with $T$, then $A L \subset L$, and $\|A y\|=\sup _{n \geq 0}\left\|T^{n} A y\right\| \leq\|A\|\|y\| \|, \forall y \in L$, so that the restriction of $A$ to $L$ is a bounded operator in $\|\cdot\|$, hence $A$ can be extended by continuity to $\widetilde{L}$. Thus, if $\lambda \in \varrho(T)$, then $A \equiv(\lambda-T)^{-1}$ induces a bounded operator on $\widetilde{L}$ which is the inverse of $\lambda-\widetilde{T}$. Therefore, $\sigma(\widetilde{T}) \subset \sigma(T)$. In particular, $\sigma(\widetilde{T}) \cap \mathbb{T}$ is countable. Since, by the assumptions, the means

$$
\frac{1}{n} \sum_{k=j}^{n-1+j} \lambda^{-k} T^{k} x
$$

converge uniformly in $j \geq 0$, to the same limit, say $z$ (see Remark 2.6), and since $T z=\lambda z$, we have $z \in \widetilde{L}$ and

$$
\sup _{j \geq 0}\left\|\frac{1}{n} \sum_{k=0}^{n-1} \lambda^{-k} T^{j+k} x-T^{j} z\right\| \rightarrow 0 \quad \text { as } n \rightarrow \infty,
$$

which implies that the means $\frac{1}{n} \sum_{k=0}^{n-1} \lambda^{-k} \widetilde{T}^{k} x$ converge in the norm of $L$. Therefore, by Proposition 2.11, $\left\{\widetilde{T}^{n} x: n \in \mathbb{Z}_{+}\right\}$is an asymptotically almost periodic sequence in $L$, which also implies that $\left\{T^{n} x: n \in \mathbb{Z}_{+}\right\}$is an asymptotically almost periodic sequence in $X$. If, in addition, the above means converge to 0 for all $\lambda \in \sigma(T) \cap \mathbb{T}$, then, again by Remark 2.6 and Proposition 2.11, $\left\|\widetilde{T}^{n} x\right\| \rightarrow 0$ as $n \rightarrow \infty$, so that $\left\|T^{n} x\right\| \rightarrow 0$ as $n \rightarrow \infty$.

The Katznelson-Tzafriri Theorem also admits the following individual variant (in both Theorem 2.14 and Corollary 2.15 below $T$ need not be even bounded).

Theorem 2.14 ([15]). Let $T$ be an arbitrary operator in $X$. If $x \in \bigcap\left\{D\left(T^{n}: n \geq 0\right\}\right.$ is such that the sequence $\left\{T^{n} x: n \in \mathbb{Z}_{+}\right\}$is bounded, and if $f=\sum_{n=0}^{\infty} a_{n} \lambda^{n} \in W(\mathbb{T})$ is a function of spectral synthesis with respect to $\sigma(T) \cap \mathbb{T}$, then $\left\|T^{n}\left(\sum_{k=0}^{\infty} a_{k} T^{k} x\right)\right\| \rightarrow 0$ as $n \rightarrow \infty$.

Pro of. Let $\widetilde{L}$ and $\widetilde{T}$ be as above. Then, by Theorem $2.10,\left\|\widetilde{T}^{n} \sum_{k=0}^{\infty} a_{k} \widetilde{T}^{k} x\right\| \rightarrow 0$, which implies the conclusion.

Corollary 2.15. Let $T$ be an arbitrary operator in $X$ such that $\sigma(T) \cap \mathbb{T} \subset\{1\}$. If $x \in$ $\bigcap\left\{D\left(T^{n}\right): n \geq 0\right\}$ and the sequence $\left\{T^{n} x: n \in \mathbb{Z}_{+}\right\}$is bounded, then $\left\|T^{n+1} x-T^{n} x\right\| \rightarrow 0$ as $n \rightarrow \infty$.

4. Strongly constrictive operators. An operator $T$ on a (real or complex) Banach space $X$ is called strongly constrictive, if there is a compact set $\Omega \subset X$ such that 
$\operatorname{dist}\left(T^{n} x, \Omega\right) \rightarrow 0$ as $n \rightarrow \infty, \forall\|x\| \leq 1$. Lasota, Li and Yorke [27] introduced this class of operators in the important particular case of the Markov operators on $L^{1}$-spaces, and proved that the iterates $\left\{T^{n} x: n \in \mathbb{Z}_{+}\right\}, x \in X$, are asymptotically periodic for every strongly constrictive Markov operator on $L^{1}(\mu)$. For arbitrary operators, $C_{0}$-semigroups, and representations of general semigroups, on arbitrary Banach spaces, this class was introduced in [45], under the name compactifying operators (resp., semigroups, representations). However, it seems that that terminology did not survive, as the main results of [45] remained unnoticed and were rediscovered by many subsequent authors (see e.g. [5], [33]). Therefore, we shall adopt the terminology of strongly constrictive operators (resp., $C_{0}$-semigroups, representations) in this article. The following result is contained in [45] as a particular case of a more general result.

THEOREM 2.16. A bounded operator $T$ on $X$ is strongly constrictive if and only if the semigroup $\left\{T^{n}: n \in \mathbb{Z}_{+}\right\}$is almost periodic and $\operatorname{dim} X_{\mathrm{b}}<\infty$.

Pr o of. First observe that, if $T$ is simultaneously strongly constrictive and isometric, then the underlying Banach space must be finite dimensional. In fact, one can assume, without loss of generality, that $\Omega$ coincides with the set of all $\omega$-limit points of $\left\{T^{n} x\right.$ : $\left.n \in \mathbb{Z}_{+}\right\},\|x\| \leq 1$. Then $\Omega$ is a compact set such that $T(\Omega) \subset \Omega$. It is well known that isometries of compact metric spaces are surjective, so that $\operatorname{dist}\left(T^{n} x, \Omega\right)=\operatorname{dist}(x, \Omega)$, $\forall x \in X,\|x\| \leq 1$. Thus, the unit ball of $X$ is contained in $\Omega$, which implies that $\operatorname{dim} X<\infty$.

Now a straightforward argument shows that if $T$ is strongly constrictive, then $\left\{T^{n}\right.$ : $\left.n \in \mathbb{Z}_{+}\right\}$is almost periodic. The restriction $T \mid X_{\mathrm{b}}$ also is strongly constrictive, and, in a suitable equivalent norm, isometric, so $\operatorname{dim} X_{\mathrm{b}}<\infty$.

If now we assume, in addition, that $X$ is a real Banach space $\left({ }^{2}\right)$ ordered by a total minihedral cone $X^{+} \subset X$ (say, $X$ is a real Banach lattice), and let $T$ be a positive operator on $X$, i.e. $T X^{+} \subset X^{+}$, then Theorem 2.16 immediately gives the following result, which is a generalization of the Lasota-Li-Yorke Theorem. Note that the proof given in [27] for Markov operators in $L^{1}(\mu)$ is very different (and more complicated) and does not seem to be suitable for such generalizations.

THEOREM 2.17. Let $T$ be a positive strongly constrictive operator on a real Banach space ordered by a total minihedral cone (in particular, on a real Banach lattice). Then there exist $\left\{e_{1}, \ldots, e_{n}\right\}$ in $X^{+},\left\{g_{1}, \ldots, g_{n}\right\}$ in $X^{*}$ such that $\left\langle e_{i}, g_{k}\right\rangle=\delta_{i k}, \forall i, k$, and a permutation $\sigma$ of $\{1, \ldots, n\}$, such that

$$
\lim _{k \rightarrow \infty}\left\|T^{k}\left(x-\sum_{i=1}^{n}\left\langle x, g_{i}\right\rangle e_{i}\right)\right\|=0, \quad \forall x \in X,
$$

and

$$
T e_{i}=e_{\sigma(i)}, \quad \forall i=1, \ldots, n
$$

$\left(^{2}\right)$ If $X$ is a real Banach space and $\left\{T^{n}: n \in \mathbb{Z}_{+}\right\}$is almost periodic, then the de LeeuwGlicksberg Decomposition is considered for the complexification of $T$. 
Proof. By Theorem 2.16, the semigroup $\left\{T^{n}: n \in \mathbb{Z}_{+}\right\}$is almost periodic with finite-dimensional boundary subspace $X_{\mathrm{b}}$. We can assume, without loss of generality, that $\|T\| \leq 1$, so that $T \mid X_{\mathrm{b}}$ is isometric. Since $T$ is positive, it follows that the boundary projection $P$ also is positive. In the boundary subspace $X_{\mathrm{b}}$ the cone $X_{\mathrm{b}}^{+}=X^{+} \cap X_{\mathrm{b}}=$ $P X^{+}$is closed and total, and since $X^{+}$is a minihedral cone, $P X^{+}$also is a minihedral cone in $X_{\mathrm{b}}$. Since $\operatorname{dim} X_{\mathrm{b}}<\infty, P X^{+}$is a solid cone. It is well known that a closed solid minihedral cone in a finite-dimensional space is the coordinate cone, i.e. $X_{\mathrm{b}}$ is algebraic and order isomorphic to $\mathbb{R}^{n}$, where $n=\operatorname{dim} X_{\mathrm{b}}$. Let $\Gamma$ be the intersection of the extreme rays of the cone $X_{\mathrm{b}}^{+}$with the unit sphere of $X_{\mathrm{b}}$. Thus, $\Gamma$ is a basis of $X_{\mathrm{b}}$. Let $\left\{e_{1}, \ldots, e_{n}\right\}$ be elements of $\Gamma$. Let $\left\langle x, g_{i}\right\rangle$ be the coordinates of the vector $x \in X_{\mathrm{b}}$ in the basis $\left\{e_{i}\right\}_{i=1}^{n}$, i.e. $g_{i}$ is a linear functional on $X_{\mathrm{b}}$, and denote by the same letter $g_{i}$ an extension of $g_{i}$ to the whole $X$ such that $g_{i} \mid X_{\mathrm{s}} \equiv 0, \forall i$. Since $T \mid X_{\mathrm{b}}$ is simultaneously an isometry and order isomorphism, it follows that $T \Gamma=\Gamma$. Therefore, there exists a permutation $\sigma:\{1, \ldots, n\} \rightarrow\{1, \ldots, n\}$ such that $T e_{i}=e_{\sigma(i)}$. It is easy to see that

$$
\left\langle x-\sum_{i=1}^{n}\left\langle x, g_{i}\right\rangle e_{i}, g_{j}\right\rangle=0, \quad \forall j=1, \ldots, n,
$$

which implies that $x-\sum_{i=1}^{n}\left\langle x, g_{i}\right\rangle e_{i} \in X_{\mathrm{s}}$, i.e. (2.2) holds.

A sequence $\left\{x_{n}: n \in \mathbb{Z}_{+}\right\}$in $X$ is called asymptotically periodic if $x_{n}=y_{n}+z_{n}$, where $y_{n}$ is periodic, and $\left\|z_{n}\right\| \rightarrow 0$ as $n \rightarrow \infty$. From Theorem 2.17 we have the following corollary.

COROLlary 2.18. Under the condition of Theorem 2.17 the sequence of iterates $\left\{T^{n} x\right\}_{n=0}^{\infty}$ is asymptotically periodic.

3. $C_{0}$-semigroups. In this section, we shall describe corresponding results for $C_{0^{-}}$ semigroups which are analogous to the results of the previous section. Throughout this section, if not otherwise stated, $T(t), t \geq 0$, is a bounded $C_{0}$-semigroup on a Banach space $X$, with generator $A$. $T(t)$ is called a bounded semigroup if $\sup _{t>0}\|T(t)\|<\infty$, and a strongly stable semigroup if $\|T(t) x\| \rightarrow 0$ as $t \rightarrow \infty, \forall x \in X$. We use the same notations for various notions of spectrum for the generator $A$. Thus, in particular, $E \sigma(A)$ is the ergodic spectrum of $A$, i.e.

$$
E \sigma(A)=\left\{\lambda \in i \mathbb{R}: \exists x^{*} \in D\left(A^{*}\right): x^{*} \neq 0, A^{*} x^{*}=\lambda x^{*}, x^{*} \mid X_{\lambda}(A) \equiv 0\right\} .
$$

1. The limit isometric semigroup. The following proposition is an analog of Proposition 2.1 .

Proposition 3.1 ([42]). Assume that the semigroup $\left\{T(t): t \in \mathbb{R}_{+}\right\}$is bounded. Then there exist another Banach space $E$, a continuous linear operator $Q: X \rightarrow E$, with dense range, and an isometric $C_{0}$-semigroup $\left\{V(t): t \in \mathbb{R}_{+}\right\}$on $E$, with generator $B$, such that:

(i) $Q T(t)=V(t) Q, \forall t \geq 0$;

(ii) $\sigma(B) \subset \sigma(A), P \sigma(A) \cap i \mathbb{R} \subset P \sigma(B), P \sigma\left(B^{*}\right) \subset P \sigma\left(A^{*}\right)$;

(iii) $\|Q x\|_{E}=\lim \sup _{n \rightarrow \infty}\|T(t) x\|$. 
Moreover, such $E$ and $V(t)$ are unique in the sense that if $E^{\prime}$ is another Banach space and $V^{\prime}(t): E^{\prime} \rightarrow E^{\prime}$ is an isometric $C_{0}$-semigroup satisfying (i)-(iii), then there is an invertible isometric operator $U: E \rightarrow E^{\prime}$ such that $U V(t) U^{-1}=V^{\prime}(t), \forall t \geq 0$.

Proof. The proof is analogous to the proof of Proposition 2.1. Thus, introducing, if necessary, the new equivalent norm $\|x\|=\sup _{n \geq 0}\|T(t) x\|$ in $X$, we can assume that $T(t)$ are contractions, $\forall t \in \mathbb{R}_{+}$. Let $l(x)$ be a semi-norm in $X$ defined by $l(x) \equiv$ $\lim _{n \rightarrow \infty}\|T(t) x\|, x \in X, \widehat{X}=X / \operatorname{ker} l$ and let $Q$ denote the natural homomorphism from $X$ to $\widehat{X}$. The semi-norm $l$ induces in the quotient space $\widehat{X}$ a norm $\widehat{l}$ by $\widehat{l}(Q x) \equiv l(x)$. Let $\widehat{T}(t): \widehat{X} \rightarrow \widehat{X}$ be defined by $\widehat{T}(t)(Q x)=Q(T(t) x), t \geq 0$. Clearly, $\widehat{T}(t)$ is a strongly continuous isometric semigroup on the normed space $(\widehat{X}, \widehat{l})$. Let $E$ be the completion of $\widehat{X}$ and let $V(t)$ be the extension of $\widehat{T}(t)$ to the whole $E$ by continuity. Then $V(t)$ is isometric, $Q$ is a bounded linear operator from $X$ to $E$ with dense range. All properties (i)-(iii) in Proposition 3.1 can be shown in the same way as in the proof of Proposition 2.1, except for the inclusion $\sigma(B) \subset \sigma(A)$, which can be shown as follows: If $\operatorname{Re} \lambda>0$, then, since $T(t)$ is a bounded semigroup, $R_{A}(\lambda) \equiv(A-\lambda)^{-1}$ exists and equals $-\int_{0}^{\infty} e^{-\lambda t} T(t) d t$. Therefore, $\widehat{R}_{A}(\lambda)(Q x)=Q\left(R_{A}(\lambda) x\right)=-\int_{0}^{\infty} e^{-\lambda t} V(t)(Q x) d t=(B-\lambda)^{-1}(Q x)$, so that $\widehat{R}_{A}(\lambda)=R_{B}(\lambda)$. Now, if $\mu \in \varrho(A)$, then from the Hilbert identity $R_{A}(\lambda)-R_{A}(\mu)=$ $(\lambda-\mu) R_{A}(\lambda) R_{A}(\mu)$, it follows that $\widehat{R}_{A}(\mu)\left[I+(\lambda-\mu) \widehat{R}_{A}(\lambda)\right]=\widehat{R}_{A}(\lambda)$, or

$$
\widehat{R}_{A}(\mu)\left[I+(\lambda-\mu) R_{B}(\lambda)\right]=R_{B}(\lambda)
$$

which implies $\widehat{R}_{A}(\mu)(B-\mu)=\widehat{R}_{A}(\mu)\left[I+(\lambda-\mu) R_{B}(\lambda)\right](B-\lambda)=I$, and analogously $(B-\mu) \widehat{R}_{A}(\mu)=I$. Thus, $\mu \in \varrho(B)$.

From Proposition 3.1 we also obtain the following characterization of strongly stable $C_{0}$-semigroups (cf. [44, Theorem 2.3]).

THEOREM 3.2. If $T(t)$ is a bounded $C_{0}$-semigroup, then the following are equivalent.

(1) $T$ is strongly stable;

(2) There does not exist a bounded function $x^{*}(t):(-\infty, 0] \rightarrow X^{*}$ such that $T^{*}(t) x^{*}(-t)=x_{0}^{*}, x_{0}^{*} \neq 0$;

(3) There does not exist a bounded function $x^{*}(t): \mathbb{R} \rightarrow X$ such that

$$
T^{*}(s) x^{*}(t)=x^{*}(t+s), \quad \forall t \in \mathbb{R}, \forall s \geq 0 .
$$

Proof. $(1) \Rightarrow(2)$. If $T(t)$ is strongly stable and if, on the contrary, there exists a bounded function $x^{*}(t):(-\infty, 0] \rightarrow X^{*}$ such that $T^{*}(t) x^{*}(-t)=x_{0}^{*} \neq 0, \forall t \geq 0$, then, for every $x \in X, 0=\lim _{t \rightarrow \infty}\left|\left\langle T(t) x, x^{*}(-t)\right\rangle\right|=\left|\left\langle x, T^{*}(t) x^{*}(-t)\right\rangle\right|=\left|\left\langle x, x_{0}^{*}\right\rangle\right|$, which is a contradiction.

$(2) \Rightarrow(3)$ is trivial.

$(3) \Rightarrow(1)$. If $T(t)$ is not strongly stable, then the limit isometric semigroup $V(t)$ constructed in Proposition 3.1 is nontrivial (i.e. $E \neq 0$ ). For a nonzero element $z_{0}^{*}$ in $E^{*}$, one can find, by Theorem 2.2 , a bounded sequence $z^{*}(n), n=0, \pm 1, \pm 2, \ldots$, in $E^{*}$ such that $V^{*}(k) z^{*}(n)=z^{*}(k+n), \forall k \geq 0, \forall n \in \mathbb{Z}$. For any $\tau \in(-n,-n+1), n=1,2, \ldots$, let $\tau=-n+1-s(0<s<1)$, and put $z^{*}(\tau)=V^{*}(1-s) z^{*}(-n), z^{*}(t)=V^{*}(t) z_{0}^{*}$, $\forall t \geq 0$. It is easy to see that $V^{*}(s) z^{*}(t)=z^{*}(s+t), \forall s \geq 0, \forall t \in \mathbb{R}$. Let $x^{*}(t), t \in \mathbb{R}_{+}$, 
be the corresponding elements of $X^{*}$ defined by $\left\langle x, x^{*}(t)\right\rangle=\left\langle Q x, z^{*}(t)\right\rangle$. Then, as can be verified directly, $x^{*}(t)$ is uniformly bounded and satisfy condition (3.1).

A function $x^{*}(t)$ satisfying (3.1) is called a complete trajectory of $T^{*}(t)$. It is easy to see that every bounded complete trajectory $x^{*}(t)$ is automatically weak* continuous. By taking $x_{\phi}(s) \equiv\left(\phi * x^{*}\right)(s)$ with a suitable function $\phi \in L^{1}(\mathbb{R})$, we see that there exist nonzero bounded uniformly weak* continuous complete trajectories. If $T^{*}(t)$ is strongly continuous, then the trajectories are uniformly strongly continuous.

2. Almost periodicity and stability. Recall that $\left\{T(t): t \in \mathbb{R}_{+}\right\}$is called an almost periodic $C_{0}$-semigroup if $\left\{T(t) x: t \in \mathbb{R}_{+}\right\}$is relatively compact, $\forall x \in X$. If $\{T(t): t \in \mathbb{R}\}$ is a group, then it is called an almost periodic group if $\{T(t) x: t \in \mathbb{R}\}$ is relatively compact, $\forall x \in X$. If $\{T(t): t \in \mathbb{R}\}$ is an almost periodic group, then $X=\overline{\operatorname{span}}\{x \in$ $\left.X: \exists \lambda \in \mathbb{R}: T(t)=e^{i \lambda t} x, \forall t \in \mathbb{R}\right\}$. If $\left\{T(t): t \in \mathbb{R}_{+}\right\}$is a strongly stable semigroup then, obviously, $\left\{T(t): t \in \mathbb{R}_{+}\right\}$is almost periodic. These are two extreme cases of almost periodic semigroups, as shown by the following theorem, known as the de LeeuwGlicksberg Decomposition for $C_{0}$-semigroups (see [26], [30]).

TheOREM 3.3. If the semigroup $\left\{T(t): t \in \mathbb{R}_{+}\right\}$is almost periodic, then $X=X_{\mathrm{s}} \dot{+} X_{\mathrm{b}}$, where $X_{\mathrm{s}}=\left\{x \in X: \lim _{t \rightarrow \infty}\|T(t) x\|=0\right\}, X_{\mathrm{b}}=\overline{\operatorname{span}}\left\{x \in X: \exists \lambda \in \mathbb{R}: T(t) x=e^{i \lambda t} x\right\}$. Moreover, $T(t) \mid X_{\mathrm{b}}$ are invertible, $\forall t \geq 0$, and generate an almost periodic group. The projection $P: X \rightarrow X_{\mathrm{b}}$, ker $P=X_{\mathrm{s}}$, belongs to the strong operator closure of $\{T(t)$ : $\left.t \in \mathbb{R}_{+}\right\}$. If , in addition, $T(t)$ is a contraction semigroup, then $T(t) \mid X_{\mathrm{b}}$ are invertible isometries and $P$ is an orthogonal projection.

As in the discrete case, $X_{\mathrm{b}}$ and $X_{\mathrm{s}}$ are called the boundary and stable subspaces, and $P$ is called the boundary projection, respectively. For an arbitrary bounded, but not necessarily almost periodic, semigroup $\left\{T(t): t \in \mathbb{R}_{+}\right\}$we can define $X_{\mathrm{b}}$ and $X_{\mathrm{s}}$ as in Theorem 3.3. They form a direct topological sum $X_{\mathrm{ap}}=X_{\mathrm{b}} \dot{+} X_{\mathrm{s}}$, and the restriction $\left\{\left(T(t) \mid X_{\text {ap }}\right): t \in \mathbb{R}_{+}\right\}$can be extended to an almost periodic group.

Using this fact, and the same method as in the proof of Proposition 2.4, we can prove the following variant of Proposition 3.1.

Proposition 3.4. Assume that the $C_{0}$-semigroup $\left\{T(t): t \in \mathbb{R}_{+}\right\}$is bounded. There exist another Banach space $F$, a continuous linear operator $R: X \rightarrow F$, with dense range, and an isometric $C_{0}$-semigroup $\left\{U(t): t \in \mathbb{R}_{+}\right\}$on $F$, with generator $C$, such that:

(i) $R T(t)=U(t) R, \forall t \geq 0$;

(ii) $\sigma(C) \subset \sigma(A), P \sigma(C)=\emptyset, P \sigma\left(C^{*}\right) \subset P \sigma\left(A^{*}\right), P \sigma\left(C^{*}\right) \cap i \mathbb{R} \subset E \sigma(A)$;

(iii) $\|R x\|_{F}=\lim \sup _{t \rightarrow \infty} \inf _{y \in X_{\mathrm{b}}}\|T(t)(x-y)\|$.

Moreover, such $F$ and $U$ are unique in the sense that if $F^{\prime}$ is another Banach space and $U^{\prime}(t): F^{\prime} \rightarrow F^{\prime}$ is an isometric $C_{0}$-semigroup satisfying (i)-(iii), then there is an invertible isometric operator $W: F \rightarrow F^{\prime}$ such that $W U(t) W^{-1}=U^{\prime}(t), \forall t \geq 0$.

The semigroup $\left\{U(t): t \in \mathbb{R}_{+}\right\}$can be called the limit isometric semigroup of $T(t)$ with continuous spectrum.

Proposition 3.4 enables us to prove the following Almost Periodicity Theorem which was obtained in [41]. 
Theorem 3.5. If the semigroup $\left\{T(t): t \in \mathbb{R}_{+}\right\}$is bounded, $\sigma(A) \cap i \mathbb{R}$ is countable and $E \sigma(A) \cap i \mathbb{R}$ is empty, then $\left\{T(t): t \in \mathbb{R}_{+}\right\}$is almost periodic.

Proof. The proof of Theorem 3.5 is analogous to that of Theorem 2.5. Assuming that $T(t)$ is not almost periodic, one can use the construction of Proposition 3.4 to obtain an isometric semigroup $U(t)$, with generator $C$, on $F \neq\{0\}$. As an intermediate step, we need to show that $U(t)$ is invertible for every $t$. This follows from the following proposition.

Proposition 3.6. If $\left\{T(t): t \in \mathbb{R}_{+}\right\}$is an isometric $C_{0}$-semigroup, with generator $A$, such that $\sigma(A) \cap i \mathbb{R}$ is a proper subset of $i \mathbb{R}$, then $T(t)$ is invertible for every $t \geq 0$, so that $T(t)$ extends to an isometric $C_{0}$-group.

Proof. First we show that, if $\operatorname{Re} \lambda<0$, then

$$
\|(\lambda-A) x\| \geq|\operatorname{Re} \lambda| \cdot\|x\|, \quad \forall x \in D(A) .
$$

In fact, for the function $u(t) \equiv e^{-\lambda t} T(t) x, t \geq 0$, we have

$$
u(t)=x+\int_{0}^{t} \frac{d u(\tau)}{d \tau} d \tau=x+\int_{0}^{t} e^{-\lambda \tau} T(\tau)(A x-\lambda x) d \tau,
$$

which implies

$$
e^{-\operatorname{Re} \lambda t}=\|u(t)\| \leq\|x\|+\frac{e^{-\operatorname{Re} \lambda t}-1}{-\operatorname{Re} \lambda}\|A x-\lambda x\|,
$$

and hence (3.2) holds.

From (3.2) it follows that all the points $\lambda$ such that $\operatorname{Re} \lambda<0$, belong to the same regular component of the operator $A$. Since there is a $\lambda_{0} \in \varrho(A) \cap i \mathbb{R}$, it follows that $\lambda \in \varrho(A)$, and $\left\|(\lambda-A)^{-1}\right\| \leq 1 /|\operatorname{Re} \lambda|$. Applying the Hille-Yosida Theorem, we see that $-A$ generates a contraction semigroup, say $S(t), t \geq 0$. Since $(d / d t)(S(t) T(t))=0$, it follows $S(t) T(t)=T(t) S(t)=I$, which completes the proof of Proposition 3.6.

Now we can continue the proof of Theorem 3.5. Since $U(t)$ can be extended to an isometric $C_{0}$-group, $\sigma(C) \neq \emptyset$ and $\sigma(C) \subset i \mathbb{R}$. By property (ii) of Proposition 3.4, $\sigma(C)$ is countable. Choose an isolated point $\lambda \in \sigma(C)$. Then $\lambda$ is an eigenvalue of $C$ and $C^{*}$, so, there is a functional $\phi \in F^{*}, \phi \neq 0$, such that $C^{*} \phi=\lambda \phi$. The functional $\varphi \in X^{*}$ defined by $\langle x, \varphi\rangle \equiv\langle Q x, \phi\rangle$ is nonzero and satisfies $A^{*} \varphi=\lambda \varphi, \varphi \mid X_{\mathrm{b}} \equiv 0$, i.e. $\lambda \in E \sigma(A)$, which is a contradiction.

Remark 3.7. Recall that the semigroup $\{T(t): t \geq 0\}$ is called ergodic if $R^{-1} \int_{0}^{R} T(t) d t$ converges strongly to $P$ (as $R \rightarrow \infty$ ), where $P$ is the projection from $X$ onto $X_{0} \equiv\{x \in X: T(t) x=x, \forall t \geq 0\}=\{x \in D(A): A x=0\}$. It is known that $i \lambda \notin E \sigma(A), \lambda \in \mathbb{R}$, if and only if $\left\{e^{-i \lambda t} T(t): t \geq 0\right\}$ is ergodic. On the other hand, if $\{T(t): t \geq 0\}$ is bounded and $R^{-1} \int_{0}^{R} T(t) x d t$ converges, then $R^{-1} \int_{a}^{R+a} T(t) x d t$ converges uniformly in $a \geq 0$; hence the restriction $\{T(t): t \geq 0\}$ to the subspace $\overline{\operatorname{span}}\{T(t) x: t \geq 0\}$ is ergodic.

Theorem 3.5 implies the following corollary, which can also be proved directly by applying the same argument and Proposition 3.1. This result was obtained independently 
by Arendt and Batty [1], with a completely different proof. Another proof has been subsequently given by Esterle, Strouse and Zouakia [18].

Corollary 3.8. If $\left\{T(t): t \in \mathbb{R}_{+}\right\}$is a bounded $C_{0}$-semigroup such that $\sigma(A) \cap i \mathbb{R}$ is countable and $\operatorname{P\sigma }\left(A^{*}\right) \cap i \mathbb{R}$ is empty, then $T(t)$ is strongly stable.

From the cited Sz.-Nagy-Foiaş Theorem it follows, by applying a standard argument involving the Cayley transform (see [34, Chapter 3]), that if $T(t)$ is a c.n.u. contraction semigroup, with generator $A$, on a Hilbert space and if $m(\sigma(A) \cap i \mathbb{R})=0$ (where $m$ is the Lebesgue measure on $i \mathbb{R})$, then $T(t)$ is strongly stable. However, the Sz.-NagyFoiaş Theorem does not apply to bounded $C_{0^{-}}$-semigroups since, in general, bounded $C_{0^{-}}$ semigroups are not similar to contractions semigroups. A corresponding counterexample has been constructed by Packel [36] (see also [11]) by modifying the Foguel's and Halmos' examples. (See also remarks in Section 6).

Theorem 2.9 also has a corresponding one-parameter analog. Recall that a function $f \in L^{1}(\mathbb{R})$ is said to be a function of spectral synthesis with respect to a closed subset $\Delta$ of $\mathbb{R}$ if there is a sequence $g_{n} \in L^{1}(\mathbb{R})$ such that $\widehat{g}_{n}$ vanishes in a neighborhood $\mathcal{U}_{n}$ of $\Delta$ and $\left\|g_{n}-f\right\|_{L^{1}} \rightarrow 0$ as $n \rightarrow \infty$. The following theorem was obtained in [42] and independently with a completely different proof in [18].

THEOREM 3.9. If $\left\{T(t): t \in \mathbb{R}_{+}\right\}$is bounded and $f \in L^{1}\left(\mathbb{R}_{+}\right)$is a function of spectral synthesis with respect to $(-i \sigma(A)) \cap \mathbb{R}$, then $\|T(t) \widehat{f}(T)\| \rightarrow 0$ as $t \rightarrow 0$.

The proof of Theorem 3.9 is analogous to that of Theorem 2.9. Namely, one can first prove by a similar argument, using Proposition 3.1 and the well known facts about isometric $C_{0}$-groups, that $\|T(t) \widehat{f}(T) x\| \rightarrow 0, \forall x \in X$ as $t \rightarrow \infty$. But now the semigroup $\widetilde{T}(t): B(X) \rightarrow B(X)$, defined by $\widetilde{T}(t) T=T(t) Y, Y \in B(X)$, is not, in general, strongly continuous, so we need to restrict it to a suitable subspace $L$ of $B(X)$ consisting of those $Y$ for which $t \mapsto T(t) Y$ is continuous from $\mathbb{R}_{+}$to $B(X)$, and apply the strong convergence version of Theorem 3.9 to the semigroup $\widetilde{T}(t) \mid L$. Theorem 3.9 gives the following corollary.

COROllary 3.10. If $T$ is a bounded $C_{0}$-semigroup and $\sigma(T) \cap i \mathbb{R} \subset\{0\}$, then $\left\|[T(t+s)-T(t)] R_{A}(\lambda)\right\| \rightarrow 0, \forall s>0$, as $t \rightarrow \infty$.

3. Behaviour of individual elements. From Theorem 3.5 and Remark 3.7 it also follows that the following holds.

Proposition 3.11. If $\left\{T(t): t \in \mathbb{R}_{+}\right\}$is a bounded $C_{0}$-semigroup such that $\sigma(A) \cap i \mathbb{R}$ is countable, and if $x$ is a vector in $X$ such that the means

$$
\frac{1}{R} \int_{0}^{R} e^{-\lambda t} T(t) x d t
$$

converge, $\forall \lambda \in \sigma(A) \cap i \mathbb{R}$, then $T(t) x$ is an asymptotically a.p. function. In particular, if the above means converge to 0 , for all $\lambda \in \sigma(A) \cap i \mathbb{R}$, then $\|T(t) x\| \rightarrow 0$ as $t \rightarrow \infty$.

P r o o f. To prove Proposition 3.11, it is enough to apply Theorem 3.5 to the restriction of $T(t)$ to $\overline{\operatorname{span}}\{T(t) x: t \geq 0\}$.

Remark 3.12. We note also the difference in the terminology. If $\{T(t): t \in \mathbb{R}\}$ is an almost periodic group, then, for each $x$ in $X$, the function $x(t) \equiv T(t) x, t \in \mathbb{R}$, 
is almost periodic, i.e. the family of translates $\left\{x_{s}(t) \equiv x(t+s), s \in \mathbb{R}\right\}$, is relatively compact in $B C(\mathbb{R}, X)$, the Banach space of bounded continuous $X$-valued functions on $\mathbb{R}$. If $\{T(t): t \geq 0\}$ is an almost periodic semigroup and $x \in X$, then, the function $x(t)=T(t) x, t \in \mathbb{R}_{+}$, is asymptotically almost periodic (i.e. there is an almost periodic function $y: \mathbb{R} \rightarrow X$ such that $\|x(t)-y(t)\| \rightarrow 0$ as $t \rightarrow \infty)$, but it need not be almost periodic (i.e. $x(t)$ may not be extendable to an almost periodic function on $\mathbb{R}$ ). Thus, almost periodic semigroups would be more exactly called asymptotically almost periodic. However, we preferred to follow the established terminology.

Proposition 3.11 allows us to prove individual variants of Theorems 3.5, 3.9 and Corollaries 3.8, 3.10 for arbitrary (in general unbounded) $C_{0}$-semigroups, as we did in Section 2 for discrete semigroups.

Moreover, we can obtain even more general results applying to the case when $A$ is not assumed to be the generator of a $C_{0}$-semigroup, and when the role of the individual trajectory $T(t) x$ is played by a bounded mild solution of the abstract differential equation

$$
x^{\prime}(t)=A x(t), \quad t \geq 0, \quad x(0)=x .
$$

This generalization is based on the following definition of the Hille-Yosida space (see [13] for details).

Let $A$ be a closed linear operator on $X$. A continuous function $u: \mathbb{R}_{+} \rightarrow X$ is called a mild solution of (3.3), if $v^{\prime}(t)=A v(t)+x$, where $v(t) \equiv \int_{0}^{t} u(s) d s$. Assume that $A$ satisfies the uniqueness condition for the Cauchy problem, i.e., all the mild solutions of (3.3) which are exponentially bounded (i.e. $\|u(t)\| \leq M e^{\omega t}$, for some $M$ and $\omega$ ) are unique. Denote by $Z_{0}$ the set of all $x \in X$ for which (3.3) has a bounded uniformly continuous mild solution $u(t, x), t \geq 0$, and let $\|\cdot\|_{Z_{0}}$ be a norm in $Z_{0}$ defined by

$$
\|x\|_{Z_{0}} \equiv \sup _{t \geq 0}\|u(t, x)\|
$$

Then, as is known (see [13]), $Z_{0}$ is a Banach space, there is a continuous embedding of $Z_{0}$ into $X$, the restriction $A \mid Z_{0}$ generates a contraction $C_{0}$-semigroup $T(t)$ defined by $T(t) x \equiv u(t, x), t \geq 0$, and $\sigma\left(A \mid Z_{0}\right) \subseteq \sigma(A)$. Moreover, if $B: X \rightarrow X$ is a bounded linear operator such that $B A \subseteq A B$, then $B$ restricted to $Z_{0}$ also is a bounded operator and $\|B\|_{Z_{0}} \leq\|B\|$.

Theorem 3.13 (deLaubenfels and Vũ Quôc Phóng [14]). Let A be an arbitrary closed linear operator on $X$. Suppose that $A$ is such that mild solutions to the abstract Cauchy problem (3.3) are unique, and that $\sigma(A) \cap i \mathbb{R}$ is countable. Let $u(t) \equiv u(t, x), t \geq 0$, be a bounded, uniformly continuous mild solution of (3.3). Then $u(t)$ is asymptotically almost periodic if and only if for every $\lambda \in \sigma(A) \cap i \mathbb{R}$, the function $t \mapsto e^{-\lambda t} u(t)$ has uniformly convergent means. If, in addition, the means converge to $0, \forall \lambda \in \sigma(A) \cap i \mathbb{R}$, then $\|u(t)\| \rightarrow 0$ as $t \rightarrow \infty$.

Pr o of. The proof consists in applying Proposition 3.11 to the semigroup $T(t)$ on the Hille-Yosida space, constructed as above. From the properties of the Hille-Yosida space it follows that $\sigma\left(A \mid Z_{0}\right) \cap i \mathbb{R}$ is countable. Since $e^{-\lambda t} u(t)$ has uniformly convergent means in the norm of $X$, it also follows that the means of this function converge in the norm of $Z_{0}$, so that all the conditions of Proposition 3.11 are fulfilled. 
Theorem 3.9 and Corollary 3.10 also admit the following individual variants (here $\widehat{u}$ denotes the Laplace transform of $u$, i.e. $\left.\widehat{u}(\lambda)=\int_{0}^{\infty} e^{-\lambda t} u(t) d t, \forall \lambda, \operatorname{Re} \lambda>0\right)$.

Theorem 3.14 ([14]). Let $A$ be as in Theorem 3.13 and $u(t) \equiv u(t, x), t \geq 0$, be a bounded, uniformly continuous mild solution of (3.3). If $f \in L^{1}\left(\mathbb{R}_{+}\right)$is a function of spectral synthesis with respect to $(-i \sigma(A)) \cap \mathbb{R}$, then $\left\|\int_{0}^{\infty} f(s) u(t+s) d s\right\| \rightarrow 0$ as $t \rightarrow 0$.

Corollary 3.15. If $\sigma(A) \cap i \mathbb{R} \subset\{0\}$, and $u(t)$ is a bounded, uniformly continuous mild solution of (3.3), then $\left\|\widehat{u}_{t+s}(\lambda)-\widehat{u}_{t}(\lambda)\right\| \rightarrow 0$ as $t \rightarrow \infty, \forall s \geq 0, \operatorname{Re} \lambda>0$.

4. Strongly constrictive $C_{0}$-semigroups. A $C_{0}$-semigroup $T(t), t \geq 0$, on $X$ is called strongly constrictive if there is a compact set $\Omega \subset X$ such that $\operatorname{dist}(T(t) x, \Omega) \rightarrow 0$ as $t \rightarrow \infty, \forall\|x\| \leq 1$. The following result, which is analogous to Theorem 2.16 (with almost the same proof), is a characterization of strongly constrictive semigroups among almost periodic semigroups.

THEOREM 3.16. A bounded $C_{0}$-semigroup $T(t)$ on $X$ is strongly constrictive if and only if it is almost periodic and has finite dimensional boundary subspace.

Assume, in addition, that $X$ is a real Banach space which is ordered by a total minihedral cone $X^{+}$(in particular, $X$ is a real Banach lattice), and that $T(t)$ is a strongly constrictive $C_{0}$-semigroup of positive operators (positive semigroup) on $X$. Then all the arguments in Section 2.4 can be applied to operators $T(t)$. Thus, the boundary subspace $X_{\mathrm{b}}$ is isomorphic to $\mathbb{R}^{n}$, and $T(t)$ acts on the basis $e_{1}, \ldots, e_{n}$ as permutations, say $\sigma_{t}$, $t \geq 0$. The continuity condition of $T(t)$ then implies that $\sigma_{t}(i)=i$ for every $t \geq 0$, $i=1, \ldots, n$. Thus, the following theorem holds, which also gives an example of a situation for continuous semigroups which can differ substantially from that of discrete semigroups.

THEOREM 3.14. If $X$ is a real Banach space ordered by a total minihedral cone (e.g., a real Banach lattice), and if $T(t)$ is a positive strongly constrictive $C_{0}$-semigroup on $X$, then $T(t)$ converges strongly to a positive finite rank projection.

4. Representation of general semigroups. The results of Sections 2 and 3 can be generalized in a suitable manner to representations of locally compact abelian semigroups. Such generalizations are non-trivial, and rely on the powerful Banach algebras techniques. Because of the limited space, we restrict ourselves to a brief description of the central results, which give spectral conditions for almost periodicity and strong stability of representations.

1. General definitions. Let $S$ be a topological abelian semigroup, and $T$ be a strongly continuous representation of $S$ by bounded linear operators on $X$, i.e. $T$ is a homomorphism of $S$ into the semigroup of endomorphisms of $X$ such that the mapping $s \mapsto T(s) x$ is continuous from $S$ to $X, \forall x \in X$. If $s \mapsto T(s)$ is continuous in the uniform operator topology, then $T$ is called a uniformly continuous representation. The representation $T$ is called bounded if $\sup _{s \in S}\|T(s)\|<\infty$. Below we denote by $S^{*}$ the semigroup of semiunitary characters of $S$, i.e. $S^{*}$ consists of complex continuous homomorphisms $\xi: S \rightarrow \mathbb{C}$ such that $|\xi(s)| \leq 1, \forall s \in S\left({ }^{3}\right)$, and we endow $S^{*}$ with the topology of pointwise con-

\footnotetext{
$\left({ }^{3}\right)$ Thus, semi-unitary characters are bounded one-dimensional representations of $S$.
} 
vergence. Furthermore, we let $S_{\mathrm{u}}^{*} \equiv\left\{\xi \in S^{*}:|\xi(s)|=1, \forall s \in S\right\}$. A character $\xi$ is called an eigenvalue of $T$ if there is a nonzero vector $x \in X$ such that $T(s) x=\xi(s) x$, $\forall s \in S$; an approximate eigenvalue of $T$, if there is a net $\left\{x_{\alpha}\right\},\left\|x_{\alpha}\right\|=1$ in $X$ such that $\left\|T(s) x_{\alpha}-\xi(s) x_{\alpha}\right\| \rightarrow 0$ uniformly for $s$ in each compact subset of $S$; an $\omega$-approximate eigenvalue of $T$, if there is a sequence $\left\{x_{n}\right\}$ in $X$ such that $\left\|T(s) x_{n}-\xi(s) x_{n}\right\| \rightarrow 0$ as $n \rightarrow \infty, \forall s \in S$. The set of all approximate eigenvalues ( $\omega$-approximate eigenvalues, eigenvalues) of $T$ is denoted by $\operatorname{Sp}_{\mathrm{a}}(T)\left(\operatorname{Sp}_{\omega \mathrm{a}}(T), \operatorname{Sp}_{\mathrm{p}}(T)\right)$ and is called the approximate point spectrum (resp., the $\omega$-approximate point spectrum, the point spectrum) of the representation $T$. If $T$ is a representation, then $T^{*}: s \mapsto T^{*}(s) \equiv(T(s))^{*}$ is a weak ${ }^{*}$ continuous homomorphism of $S$ to $B\left(X^{*}\right)$, but is not, in general, a representation, as strong continuity may fail. But we denote by $\operatorname{Sp}_{\mathrm{p}}\left(T^{*}\right)$ the set of eigenvalues of $T^{*}$, i.e. characters $\xi \in S^{*}$ for which there is $\phi \in X^{*}, \phi \neq 0$, such that $T^{*}(s)=\xi(s) \phi$. Let $\operatorname{Sp}_{\mathrm{e}}(T)$ denote the set of $\xi \in S_{\mathrm{u}}^{*}$ for which there is a nonzero functional $\phi \in X^{*}$ such that $T^{*}(s) \phi=\xi(s) \phi, \forall s \in S$, and $\langle x, \phi\rangle=0$ whenever $T(s) x=\xi(s) x, \forall s \in S$. The set $\operatorname{Sp}_{\mathrm{e}}(T)$ is called the ergodic spectrum of $T$. Note that, by our definition, the ergodic spectrum always is contained in $S_{\mathrm{u}}^{*}$. If $X$ is reflexive, then $\operatorname{Sp}_{\mathrm{e}}(T)$ is empty.

Since $S$ is an abelian semigroup, we can regard $S$ as being ordered by: $s \prec t$ if there is $u \in S$ such that $s+u=t$, and we can therefore speak about convergence through $S$. For a bounded representation $T$ of $S$, we put $X_{\mathrm{b}}=\left\{x \in X: \exists \xi \in S_{\mathrm{u}}^{*}: T(s) x=\xi(s) x, \forall s \in S\right\}$, $X_{\mathrm{s}} \equiv\left\{x \in X: \lim _{S}\|T(s) x\|=0\right\}$. Clearly $X_{\mathrm{s}}$ and $X_{\mathrm{b}}$ are closed invariant subspaces under $T(s), s \in S$. The representation $T$ is called almost periodic if the set $\gamma(x) \equiv$ $\{T(s) x: s \in S\}$ is relatively compact for every $x \in X$. It is called asymptotically almost periodic if the net $\{T(s)\}_{s \in S}$ is strongly asymptotically relatively compact, i.e. for each net $\left(t_{\alpha}\right) \subset S$ such that $t_{\alpha} \rightarrow \infty$, there is a subnet $\left(s_{\beta}\right)$ such that $\lim _{\beta} T\left(s_{\beta}\right) x$ exists for each $x \in X$. In particular, if $\lim _{S}\|T(s) x\|=0, \forall x \in X$, then $T$ is called strongly stable. This class of asymptotically almost periodic representations was introduced by M. Yu. and Yu. I. Lyubich (see [30]). Note that every almost periodic representation is asymptotically almost periodic, but the converse is not true, in general (see [30]). For asymptotically almost periodic representations the following de Leeuw-Glicksberg Decomposition Theorem holds.

Theorem 4.1 (M. Lyubich and Yu. Lyubich [30]). If $T(s), s \in S$, is an asymptotically almost periodic representation, then $X=X_{\mathrm{s}} \dot{+} X_{\mathrm{b}}$. The restrictions $T(s) \mid X_{\mathrm{b}}$ are invertible and $\left\{T(s),(T(s))^{-1}: s \in S\right\}$ form a bounded almost periodic group $\left({ }^{4}\right)$. Moreover, if $T(s)$ is a contraction representation, i.e. $\|T(s)\| \leq 1, \forall s \in S$, then $T(s) \mid X_{\mathrm{b}}$ generate an isometric group, and the projection operator $P: X \rightarrow X_{\mathrm{b}}$, $\operatorname{ker} P=X_{\mathrm{s}}$, is orthogonal, i.e. $\|P\|=1$ if $P \neq 0$.

2. Almost periodicity of uniformly continuous representations. Lyubich and the author [40] obtained the following result which gives a spectral condition for asymptotic almost periodicity of a uniformly continuous representation of an arbitrary topological abelian semigroup.

$\left({ }^{4}\right)$ If $G$ is a group or semigroup of operators then $G$ is called almost periodic if the trivial representation $T: G \rightarrow B(X)(T(g)=g \forall g \in G)$ is almost periodic. 
THEOREM 4.2. If $T(s), s \in S$, is a uniformly continuous representation such that $\mathrm{Sp}_{\mathrm{a}}(T) \cap S_{\mathrm{u}}^{*}$ is countable and $\mathrm{Sp}_{\mathrm{e}}(T)$ is empty, then $T$ is asymptotically almost periodic.

Proof. We sketch the main steps of the proof, referring the reader to the original paper [40] for details.

We shall use the following facts which can be found in [30]:

(i) If $T$ is a uniformly continuous representation of a topological abelian semigroup, then $\operatorname{Sp}_{\mathrm{a}}(T)$ is nonempty.

This fundamental result, known as the Theorem on Nonemptieness of Spectrum, is due to Lyubich [28].

(ii) If $T(s), s \in S$, is a uniformly continuous representation, and $\lambda \in A \sigma\left(T\left(t_{0}\right)\right)\left({ }^{5}\right)$ for some $t_{0} \in S$, then there is $\xi \in \mathrm{Sp}_{\mathrm{a}}(T)$ such that $\xi\left(t_{0}\right)=\lambda$.

This is the Extension Theorem, also due to Lyubich. Note that (ii) can be regarded as a Spectral Mapping Theorem for uniformly continuous representations (if $S=\mathbb{R}_{+}, T(t)$, $t \geq 0$, is a uniformly continuous one-parameter semigroup, with generator $A$, then (ii) reduces to the well known Spectral Mapping Theorem: $\left.\sigma\left(e^{t A}\right)=e^{t \sigma(A)}, \forall t \geq 0\right)$.

(iii) If $T(s)$ is a uniformly continuous representation, $\mathcal{A}_{T}$ is the Banach algebra generated by $T(s), s \in S$, and $\widehat{\mathcal{A}}_{T}$ is the Gelfand space (maximal ideal space) of $\mathcal{A}_{T}$, then $\widehat{\mathcal{A}}_{T}$ can be regarded as a compact subset of $S^{*}$, and is called the $\delta$-spectrum of $T$, denoted by $\operatorname{Sp}_{\delta}(T)$. If $\xi \in \operatorname{Sp}_{\delta}(T) \cap S_{\mathrm{u}}^{*}$, then $\xi \in \operatorname{Sp}_{\mathrm{a}}(T)$.

(iv) If $L \subset X$ is an invariant subspace of a bounded representation $T$, and $\widehat{T}$ is the induced representation on $\widehat{X}=X / L$, then $\operatorname{Sp}_{\mathrm{a}}(\widehat{T}) \cap S_{\mathrm{u}}^{*} \subset \operatorname{Sp}_{\mathrm{a}}(T)$. Indeed, if $\xi \in$ $\operatorname{Sp}_{\mathrm{a}}(\widehat{T}) \cap S_{\mathrm{u}}^{*}$, then $\xi \in \operatorname{Sp}_{\delta}(\widehat{T}) \cap S_{\mathrm{u}}^{*} \subset \operatorname{Sp}_{\delta}(T) \cap S_{\mathrm{u}}^{*} \subset \operatorname{Sp}_{\mathrm{a}}(T)$, by (iii).

First of all, note that we can assume, by introducing a suitable equivalent norm, that $\|T(s)\| \leq 1, \forall s \in S$. Let $L$ be spanned by $X_{\mathrm{s}}$ and $X_{\mathrm{b}}$.

Then, by Theorem 4.1, $L=X_{\mathrm{s}} \dot{+} X_{\mathrm{b}}$. Assuming that $T$ is not asymptotically almost periodic, we have $L \neq X$. In the quotient space $\widehat{X}=X / L(\neq\{0\})$, we can consider the standard quotient topology and the induced operators $\widehat{T}(s)\left(\widehat{T}(s)(\widehat{x}) \equiv(T(s) x)^{\wedge}\right)$, which is a uniformly continuous representation of $S$ by bounded linear operators on $\widehat{X}$. Together with it we can introduce a new norm $\widehat{l}$ in $\widehat{X}$ by

$$
\widehat{l}(\widehat{x})=\lim _{S} \inf _{y \in X_{\mathrm{b}}}\|T(s)(x-y)\| .
$$

The operators $\widehat{T}(s)$ are isometric in the norm $\widehat{l}$. Let $E$ be the completion of $(\widehat{X}, \widehat{l})$ and $V(s)$ be extensions of $\widehat{T}(s)$ to the whole space $E$ by continuity. Clearly, $V(s)$ is a strongly continuous representation of $S$, but, in general, $V(s)$ is not uniformly continuous.

Consider the semigroup of isometries $\mathcal{S} \equiv\{V(s): s \in S\}$. It is a topological abelian semigroup, and it defines a trivial representation $\widetilde{V}: \mathcal{S} \rightarrow B(E)$ by $\widetilde{V}(Y)=Y, \forall Y \in \mathcal{S}$. Therefore, one can define $\operatorname{Sp}_{\mathrm{a}}(\widetilde{V})$. If $\widetilde{\xi} \in \mathcal{S}^{*}$, then $\xi(s) \equiv \widetilde{\xi}(V(s)), s \in S$, is not, in general,

$\left({ }^{5}\right)$ Here $A \sigma(T)$ denotes the approximate point spectrum of $T$, i.e. $A \sigma(T)=\{\lambda \in \mathbb{C}$ : $\exists x_{n},\left\|x_{n}\right\|=1 \forall n$, such that $\left.\lim _{n \rightarrow \infty}\left\|T x_{n}-\lambda x_{n}\right\|=0\right\}$. 
a character on $S$, as the continuity property may fail. But we show that $\operatorname{Sp}_{\mathrm{a}}(\widetilde{V}) \subset \operatorname{Sp}_{\mathrm{a}}(\widehat{T})$, i.e. if $\widetilde{\xi} \in \operatorname{Sp}_{\mathrm{a}}(\widetilde{V})$, then $\xi(s) \equiv \widetilde{\xi}\left(V(s) \in S^{*}\right.$ and $\xi \in \mathrm{Sp}_{\mathrm{a}}(\widehat{T})$.

Let $\widetilde{\xi} \in \operatorname{Sp}_{\mathrm{a}}(\widetilde{V})$ and $\left\{z_{\alpha}\right\}$ be a net in $E$ such that $\widehat{l}\left(V(s) z_{\alpha}-\widetilde{\xi}(V(s)) z_{\alpha}\right) \rightarrow 0$. Since $\widehat{X}$ is dense in $E$, one can assume that $z_{\alpha} \equiv \widehat{x}_{\alpha} \in \widehat{X}, \widehat{l}\left(z_{\alpha}\right)=1$. Hence

$$
\lim _{\alpha} \lim _{t \in S} \inf _{y \in X_{\mathrm{b}}}\left\|T(t)\left(T(s) x_{\alpha}-\xi(s) x_{\alpha}\right)-y\right\|=0, \quad \forall s \in S
$$

therefore

$$
\lim _{\alpha} \lim _{t \in S} \inf _{y \in L}\left\|T(t)\left(T(s) x_{\alpha}-\xi(s) x_{\alpha}\right)-y\right\|=0, \quad \forall s \in S
$$

i.e.

$$
\lim _{\alpha} \lim _{t \in S}\left\|\widehat{T}(t) \widehat{T}(s) z_{\alpha}-\xi(s) \widehat{T}(t) z_{\alpha}\right\|=0, \quad \forall s \in S .
$$

Since $\left\|\widehat{T}(t) z_{\alpha}\right\| \geq \widehat{l}\left(\widehat{T}(t) z_{\alpha}\right)=\widehat{l}\left(z_{\alpha}\right)=1$, it follows that $\xi(s)$ is continuous and $\xi \in \operatorname{Sp}_{\mathrm{a}}(\widehat{T})$.

From (iv) it follows that $\operatorname{Sp}_{\mathrm{a}}(\widehat{T}) \cap S_{\mathrm{u}}^{*}$ is countable, hence $\operatorname{Sp}_{\mathrm{a}}(\widetilde{V})$ is countable. By (ii) it follows that $V(s)$ is invertible, $\forall s \in S$. Therefore, $\operatorname{Sp}_{\delta}(\widetilde{V}) \subset S_{\mathrm{u}}^{*}$, hence, by (iii), $\operatorname{Sp}_{\mathrm{a}}(\widetilde{V})=\operatorname{Sp}_{\delta}(\widetilde{V})$.

Thus, the Gelfand space $\widehat{\mathcal{A}}_{T}$ of the Banach algebra $\mathcal{A}_{T}$ is countable. Since it is compact, there is an isolated point $\xi_{0}$ in $\widehat{\mathcal{A}}_{T}$. By Shilov's Idempotent Theorem, there is a projection $P$ such that $P V(s)=V(s) P, \forall s \in S$, and $\operatorname{Sp}_{\delta}(\widetilde{V} \mid(P E))=\left\{\xi_{0}\right\}$. By the Gelfand Theorem, there exists a functional $\phi \in E^{*}$ such that $V(s) \phi=\xi_{0}(s) \phi, \phi \neq 0$. Define $\varphi \in X^{*}$ by $\langle x, \varphi\rangle=\langle\widehat{x}, \phi\rangle, \forall x \in X$. Then $\varphi \mid L \equiv 0, T^{*}(s) \varphi=\xi_{0}(s) \varphi, \forall s \in S$. Since $\operatorname{Sp}_{\mathrm{e}}(T)$ is empty, it follows that there exists $x \in X$ such that $T(s) x=\xi_{0}(s) x$ and $\langle x, \varphi\rangle \neq 0$, which is a contradiction.

From Theorem 4.2 we immediately get the following corollary.

COROLlARY 4.3. If $T$ is a uniformly continuous representation of a topological Abelian semigroup $S$ such that $\operatorname{Sp}_{\mathrm{a}}(T) \cap S_{\mathrm{u}}^{*}$ is countable and $\mathrm{Sp}_{\mathrm{p}}\left(T^{*}\right) \cap S_{\mathrm{u}}^{*}$ is empty, then $T$ is strongly stable, i.e. $\lim _{S}\|T(s) x\|=0, \forall x \in X$.

Theorem 4.2 and Corollary 4.3, of course, contain as a particular case Theorem 2.5 and Corollary 2.7, as well as the corresponding results for $n$ commuting power-bounded operators and uniformly continuous one-parameter semigroups. Without additional assumptions on the semigroup $S$ the statement of Theorem 4.2 does not hold for strongly continuous representations. Indeed, if it were true, it would imply as a corollary that every strongly continuous isometric representation of a topological semigroup has nonempty spectrum, but an example is contained in [30] such that $S=G$ is a topological, not locally compact, abelian group and $T$ is a representation of $S$ by unitary operators on a Hilbert space with $\operatorname{Sp}(T)=\emptyset$. On the other hand, it is natural to expect that Theorem 4.2 can be generalized to a wide class of strongly continuous representations of suitable locally compact abelian semigroups which include $\mathbb{R}_{+}^{n}$. This question has been solved by Batty and the author [9] and is discussed below.

3. The case of strongly continuous representations. Let $G$ be a locally compact abelian group with the Haar measure $m, S$ be a subsemigroup of $G$ such that $S$ is measurable and $S-S=G$. We shall consider $S$ as a measure space with the restriction of the 
Haar measure $m$, and put $\widehat{f}(\xi) \equiv \int_{S} f(s) \xi(s) d m(s), \xi \in S^{*}$, for a function $f$ in $L^{1}(S)$. We assume that the functions $\widehat{f}(\xi), f \in L^{1}(S)$, separate points of $S^{*}$ (for example, the interior Int $S$ of $S$ is dense in $S$ ). Obviously, we can identify $S_{\mathrm{u}}^{*}$ with $G^{*}$, the dual group of unitary characters of $G$. For a bounded, strongly continuous, representation $T$ of $S$ we put $\widehat{f}(T) \equiv \int_{S} f(s) T(s) d m(s)$ and define, together with the point spectrum, the approximate point spectrum, and the $\omega$-approximate point spectrum as in Section 4.1, also the spectrum $\operatorname{Sp}(T)$ of $T$ by

$$
\operatorname{Sp}(T)=\left\{\xi \in S^{*}:|\widehat{f}(\xi)| \leq\|\widehat{f}(T)\|, \forall f \in L^{1}(S)\right\} .
$$

If $T$ is a representation of a locally compact abelian group (i.e. $S=G$ ), then $\operatorname{Sp}(T)$ concides with the $L$-spectrum introduced by Lyubich, Matsaev and Fel'dman [29], or the Arveson spectrum [3]. It is shown in [9, Proposition 2.2], that $\operatorname{Sp}_{\mathrm{a}}(T), \operatorname{Sp}_{\omega \mathrm{a}}(T)$ and $\operatorname{Sp}_{\mathrm{p}}(T)$ are contained in $\operatorname{Sp}(T)$. Moreover, $\operatorname{Sp}(T) \cap S_{\mathrm{u}}^{*} \subset \mathrm{Sp}_{\mathrm{a}}(T)$. Therefore, in the formulation of the main result in this section, Theorem 4.4, it does not matter at all whether we consider the spectrum or the approximate point spectrum of $T$. The advantage of $\operatorname{Sp}(T)$ is that it can be identified with the Gelfand space of an associated Banach algebra, which allows one to use further the powerful Banach algebras techniques.

THEOREM 4.4. If $T$ is a bounded representation of $S$ such that $\mathrm{Sp}(T) \cap S_{\mathrm{u}}^{*}$ is countable and $\mathrm{Sp}_{\mathrm{e}}(T)$ is empty, then $T$ is asymptotically almost periodic.

Proof. We shall sketch the main steps in the proof, referring the reader to [9] for more details. The proof uses some ideas and constructions some of which have already been used in the proofs of Theorems 2.5, 3.5 and 4.2. We briefly describe these facts below:

(a) (See [42]). If $T$ is a bounded representation of $S$ on $X$, then there is another Banach space $E$, a continuous linear operator $Q: X \rightarrow E$ with a dense range, and a representation $V$ of $S$ by isometric operators on $E$ such that: (i) $Q T(s)=V(s) Q$, $\forall s \in S$; (ii) $\operatorname{Sp}(V) \subset \operatorname{Sp}(T), \operatorname{Sp}_{\mathrm{p}}(T) \cap S_{\mathrm{u}}^{*} \subset \operatorname{Sp}_{\mathrm{p}}(V), \operatorname{Sp}_{\mathrm{p}}\left(V^{*}\right) \subset \operatorname{Sp}_{\mathrm{p}}\left(T^{*}\right)$; (iii) $\|Q x\|_{E}=$ $\lim \sup _{S}\|T(s) x\|, \forall x \in X$. This contruction is trivial (i.e. $E=\{0\}$ ) if, and only if, $T$ is strongly stable.

(b) If $T$ is a bounded representation of $S$ on $X$, then there is another Banach space $F$, a continuous linear operator $R: X \rightarrow F$ with a dense range, and a representation $U$ of $S$ by isometric operators on $F$ such that: (i) $R T(s)=U(s) R, \forall s \in S$; (ii) $\operatorname{Sp}(U) \subset \operatorname{Sp}(T)$, $\operatorname{Sp}_{\mathrm{p}}(U)=\emptyset, \operatorname{Sp}_{\mathrm{p}}\left(U^{*}\right) \cap S_{\mathrm{u}}^{*} \subset \operatorname{Sp}_{\mathrm{e}}(T)$; (iii) $\|R x\|_{F}=\limsup _{S} \inf _{y \in X_{\mathrm{b}}}\|T(s)(x-y)\|$, $\forall x \in X$. This contruction is trivial (i.e. $F=\{0\}$ ) if, and only if, $T$ asymptotically almost periodic.

(c) (See [9]). If $\mathcal{A}_{T}$ is the Banach algebra generated by $\widehat{f}(T), f \in L^{1}(S)$, then there is a natural bijective correspondence between $\operatorname{Sp}(T)$ and $\widehat{\mathcal{A}}_{T}$, the Gelfand space of $\mathcal{A}_{T}$. This correspondence is given by $\phi(\widehat{f}(T))=\widehat{f}(\xi), \xi \in \mathrm{Sp}(T), \phi \in \widehat{\mathcal{A}}_{T}, f \in L^{1}(S)$. In the sequel, we shall identify $\xi$ with $\phi$. In this identification, the Shilov boundary $\Gamma_{T}$ of $\mathcal{A}_{T}$ is a subset of $\operatorname{Sp}_{\mathrm{a}}(T)$. This latter fact was proved in [9] by using Żelazko's Theorem [49]. Since $\mathcal{A}_{T}$ does not in general contain a unit, we shall write $\widetilde{\mathcal{A}}_{T}$ for $\mathcal{A}_{T}+\mathbb{C} \times I$, the Banach algebra obtained from $\mathcal{A}_{T}$ by joining the unit. 
(d) (See [29], [30], [3]). If $T$ is a representation of $G$ by invertible isometries on $X$, then $\operatorname{Sp}(T) \neq \emptyset$. Moreover, if $f \in L^{1}(S)$ is such that $\widehat{f}$ vanishes on a neighborhood of $\operatorname{Sp}(T)$ then $\widehat{f}(T)=0$.

(e) If $T$ is a representation of $S$ by isometries on $X$, then $\operatorname{Sp}(T)$ is nonempty. This result on nonemptieness of spectrum of strongly continuous isometric representations can be deduced from (d) and a Banach algebra construction of Arens [2], or from (d) and a result of Douglas [16] (see [6], [9]).

Now let us outline the proof. Assuming the contrary, there is a Banach space $F$ and a representation $U$ of $S$ by isometric operators on $F$ which satisfy the properties (i)-(iii) in (b). By (e), $\operatorname{Sp}(U) \neq \emptyset$. By (c), we can identify $\operatorname{Sp}(U)$ with $\widehat{\mathcal{A}}_{U}$, so the algebra $\mathcal{A}_{U}$ is not radical, hence $\Gamma_{U}$ is nonempty. Since $\Gamma_{U} \subset \operatorname{Sp}(U) \cap S_{\mathrm{u}}^{*}$, it is a nonempty closed countable set so there exists an isolated element $\xi_{0}$ in $\Gamma_{U}, \xi_{0} \in S_{\mathrm{u}}^{*}$. By [48], $\xi_{0}$ also is isolated in $\widehat{\mathcal{A}}_{U}$.

Now we can use Shilov's Idempotent Theorem as in the last step in the proof of Theorem 4.2. By this theorem, there exists a projection $P$ in $\widetilde{\mathcal{A}}_{U}$, such that $\left(P \widetilde{\mathcal{A}}_{U}\right)^{\wedge}=$ $\left\{\xi_{0}\right\}$. Again by the Gelfand Theorem, there is a functional $\phi \in F^{*}, \phi \neq 0$, such that $U^{*}(s) \phi=\xi_{0}(s) \phi$, hence $\operatorname{Sp}_{\mathrm{e}}(T) \neq \emptyset$ (by (b)(ii)), which is a contradiction.

From Theorem 4.4, and from the de Leeuw-Glicksberg Decomposition (Theorem 4.1), we obtain the following corollary which can also be directly proved by applying the same argument (using the construction (a) instead of (b)).

THEOREM 4.5. If $T$ is a bounded representation of $S$ such that $\operatorname{Sp}(T) \cap S_{\mathrm{u}}^{*}$ is countable and $\operatorname{Sp}_{\mathrm{p}}\left(T^{*}\right) \cap S_{\mathrm{u}}^{*}$ is empty, then $T$ is strongly stable.

Using the same method, one can also prove the following generalization of Theorems 2.9 and 3.9. This generalization was obtained in [42] for uniformly continuous representations and in [9] for strongly continuous representations.

THEOREM 4.6. If $T$ is a bounded representation of $S$ on $X$ and $f \in L^{1}(S)$ is a function of spectral synthesis with respect to the set $\operatorname{Sp}(T) \cap S_{\mathrm{u}}^{*}$, then $\lim _{S}\|\widehat{f}(T) T(s)\|=0$.

5. Stability and invariant subspaces. Corollary 2.7 offered a partial answer to a general stability problem that can be formulated as follows: Find a spectral type condition that implies that the semigroup $\left\{T^{n}: n \in \mathbb{Z}_{+}\right\}$is strongly stable. Though it is not to be expected that a single spectral condition for strong stability exists for all operators, there are classes of operators in which this problem may be solved.

The stability problem is closely related to the following well known invariant subspace problem which is still unsolved (see e.g. [10], [25]): given a power-bounded operator $T$ on a Banach space $X$ such that $\left\{T^{n}: n \in \mathbb{Z}_{+}\right\}$is not strongly stable, does $T$ have a nontrivial invariant subspace? Corollary 2.7 implies that if $\sigma(T) \cap \mathbb{T}$ is countable and $\left\{T^{n}: n \in \mathbb{Z}_{+}\right\}$ is not strongly stable, then $T^{*}$ has an eigenvector so that $T^{*}$, and hence $T$, has a nontrivial invariant subspace.

From Theorem 2.2 it follows that if the semigroup $\left\{T^{n}: n \in \mathbb{Z}_{+}\right\}$is not strongly stable, then there is a bounded sequence $\left\{x_{n}^{*}: n \in \mathbb{Z}\right\}$ such that 


$$
\left(T^{*}\right)^{n} x_{k}^{*}=x_{n+k}^{*}, \quad \forall n \in \mathbb{Z}_{+}, k \in \mathbb{Z} .
$$

Condition (5.1) means that the semi-orbit $\left\{x_{n}^{*} \equiv\left(T^{*}\right)^{n} x_{0}^{*}: n \in \mathbb{Z}_{+}\right\}$can be extended to a complete orbit $\left\{x_{n}^{*}: n \in \mathbb{Z}\right\}$.

For a bounded sequence $\mathbf{y} \equiv\left\{y_{n}: n \in \mathbb{Z}\right\}$ in a Banach space $Y$, we can define the Carleman transform $\widetilde{y}(\lambda)$ by

$$
\widetilde{y}(\lambda)= \begin{cases}\sum_{k=1}^{\infty} \lambda^{k-1} y_{k} & \text { if }|\lambda|<1, \\ -\sum_{k=-\infty}^{0} \lambda^{k-1} y_{k} & \text { if }|\lambda|>1,\end{cases}
$$

and define $\operatorname{Sp}(\mathbf{y})$, the spectrum of $\mathbf{y}$, as the set of points $\lambda \in \mathbb{T}$ such that $\widetilde{y}$ does not have an analytic continuation into a neighborhood of $\lambda$.

We define the local spectrum, denoted by $\operatorname{Sp}\left(x_{0}^{*}\right)$, of the vector $x_{0}^{*}$ as the spectrum of the sequence $\left\{x_{n}^{*}: n \in \mathbb{Z}\right\}$ satisfying (5.1) (if such a sequence exists). This definition is correct if it is independent of the extensions of $\left\{x_{n}^{*}=\left(T^{*}\right)^{n} x_{0}^{*}: n \in \mathbb{Z}_{+}\right\}$for negative $n$ such that (5.1) holds, in particular, if such an extension is unique. It is easy to see that this is the case if one of the following conditions holds: (i) $T$ has a dense range (since in this case the extension is unique); (ii) $\mathbb{T} \not \subset \sigma(T)$ (since in this case the spectrum always is a proper subset of $\mathbb{T}$, and by general properties of analytic functions); or (iii) $T^{*}$ has the single-valued extension property defined below.

Since we are interested in the existence of invariant subspaces, we can always assume that the range of $T$ is dense, so that there is no ambiguity in the definition of $\operatorname{Sp}\left(x_{0}^{*}\right)$. One can show that $\operatorname{Sp}\left(x_{0}^{*}\right)$ is nonempty, $\operatorname{Sp}\left(x_{0}^{*}\right) \subset \sigma\left(T^{*}\right) \cap \mathbb{T}$, and that for every closed nonempty subset $\Lambda \subset \operatorname{Sp}\left(x_{0}^{*}\right)$ there is $y^{*} \in X^{*}, y^{*} \neq 0$, such that $\operatorname{Sp}\left(y^{*}\right) \subset \Lambda$ (cf. [44, Propositions 3.4-3.7, Section 5]). Choosing a suitable $z_{0}^{*}$ from $E^{*}$ (see the proof of Theorem 2.2 and [44, Remark 3.6]), we can achieve that $\Lambda \equiv \operatorname{Sp}\left(x_{0}^{*}\right)$ is a proper subset of $\sigma\left(T^{*}\right) \cap \mathbb{T}$. Let $M^{*}(\Lambda) \subset X^{*}$ be a linear manifold defined by $M^{*}(\Lambda)=\left\{x^{*} \in X^{*}\right.$ : there is a bounded sequence $\left\{x_{n}^{*}: n \in \mathbb{Z}\right\} \subset X^{*}, x^{*}=x_{0}^{*}$ such that (5.1) holds and $\left.\operatorname{Sp}\left(x^{*}\right) \subset \Lambda\right\}$. Clearly, $M^{*}(\Lambda)$ is invariant under $T^{*}$; moreover, it is hyperinvariant, i.e. it is invariant under every operator which commutes with $T^{*}$, but the main difficulty in proving the existence of a nontrivial invariant subspace for $T^{*}$ consists in showing that $M^{*}(\Lambda)$ is closed.

At this point, one can see a connection with the local spectral theory (see e.g. [12], $[17])$. Assume that $T^{*}$ has the single-valued extension property (SVEP), i.e., for every $x^{*} \in$ $X^{*}$, if $f(\lambda)$ and $g(\lambda)$ are $X^{*}$-valued analytic functions with domains $D(f), D(g)$ such that $D(f) \cap D(g) \supset \varrho\left(T^{*}\right)$ and $\left(\lambda-T^{*}\right) f(\lambda)=x^{*}, \forall \lambda \in D(f),\left(\lambda-T^{*}\right) g(\lambda)=x^{*}, \forall \lambda \in D(g)$, then $f(\lambda)=g(\lambda), \forall \lambda \in D(f) \cap D(g)$. Then one can define (another, different from the one just defined above) local spectrum of $x^{*}$, denoted by $\sigma\left(x^{*}\right)$, as the complement to the maximal domain of the analytic functions $f$ such that $\left(\lambda-T^{*}\right) f(\lambda)=x^{*}, \forall \lambda \in D(f)$. For a closed subset $F \subset \mathbb{C}$, let $X^{*}(F) \equiv\left\{x^{*} \in X^{*}: \sigma\left(x^{*}\right) \subset F\right\}$. Then $X^{*}(F)$ is hyperinvariant under $T^{*}$, but, in general, it is neither a nontrivial, nor a closed subspace. The operator $T^{*}$ is said to have property $(k)$ if it has the SVEP and if $X^{*}(F)$ is closed for every closed subset $F$ (see e.g. [17]). It is easy to see that if $x^{*} \in M^{*}(\Lambda)$, then $\operatorname{Sp}\left(x^{*}\right)=\sigma\left(x^{*}\right)$. Choosing a suitable closed subset $F \subset \mathbb{C}$ and a suitable $x^{*}$ in $X^{*}$ such 
that $\operatorname{Sp}\left(x^{*}\right) \cap F$ is empty, we obtain a nontrivial invariant subspace $X^{*}(F)$. Therefore, the following proposition holds.

Proposition 5.1. Assume that $T$ is a power-bounded operator on $X$ such that $T^{*}$ has property $(k)$. If $T$ is not strongly stable, then either $T$ is a scalar, or there is a closed subset $F \subset \mathbb{T}$ such that $X^{*}(F)$ is a nontrivial hyperinvariant subspace of $T^{*}$.

An operator $T$ on a Hilbert space is called hyponormal if $T^{*} T-T T^{*} \geq 0$, and cohyponormal if $T^{*}$ is hyponormal. Since it is known that every hyponormal operator $T$ has property $(k)$ [37], and that hyponormal operators whose spectrum is contained in $\mathbb{T}$ are unitary, Proposition 5.1 immediately implies the following corollary which was first obtained by Putnam [38] by a different method.

Corollary 5.2. If $T$ is a cohyponormal contraction such that $\left\{T^{n}: n \in \mathbb{Z}_{+}\right\}$is not strongly stable, then either $T$ is a scalar, or there is a closed subset $F \subset \mathbb{T}$ such that $X(F)$ is a nontrivial hyperinvariant subspace for $T^{*}$. In particular, if $T$ is a cohyponormal c.n.u. contraction, then $\left\{T^{n}: n \in \mathbb{Z}_{+}\right\}$is strongly stable.

6. Some problems. Below we formulate some open problems some of which can be regarded as steps toward a better understanding of the stability and the invariant subspace problems.

The following problems 1-3 are formulated for single operators, but analogous problems are open also for one-parameter semigroups and for (general) representations.

Problem 1. A contraction $T$ on a Banach space $X$ is called completely nonisometric (c.n.i), if there is no invariant subspace $M \subset X$ of $T$ such that $T \mid M$ is isometric.

Let $T$ be a contraction on $X$ such that $T^{*}$ is c.n.i. and $m(\sigma(T) \cap \mathbb{T})=0$. Is $\left\{T^{n}: n \in\right.$ $\left.\mathbb{Z}_{+}\right\}$strongly stable?

A partial question is: if $T^{*}$ is c.n.i. and $\sigma(T) \cap \mathbb{T}$ is a Helson set, is $\left\{T^{n}: n \in \mathbb{Z}_{+}\right\}$ strongly stable? A related question is: assuming that $T$ is power-bounded, $\left\{T^{n}: n \in \mathbb{Z}_{+}\right\}$ is not strongly stable and $\sigma(T) \cap \mathbb{T}$ is a null set or a Helson set, does $T$ have a nontrivial invariant subspace?

Problem 2. Let $H$ be a Hilbert space and $T$ be a power-bounded operator on $H$ such that $m(\sigma(T) \cap \mathbb{T})=0$. Assume that there does not exist an invariant subspace $K \subset H$ such that $T K=K, T \mid K$ is invertible and $(T \mid K)^{-1}$ is power-bounded. Is $\left\{T^{n}: n \in \mathbb{Z}_{+}\right\}$ strongly stable? A related question is: assume that $T$ is power-bounded, $\left\{T^{n}: n \in \mathbb{Z}_{+}\right\}$is not strongly stable and $\sigma(T) \cap \mathbb{T}$ is a null set, does $T$ have a nontrivial invariant subspace?

The positive solution to Problem 2 would be an extension of the Sz.-Nagy-Foiaş Theorem to power-bounded operators (cf. Remark 2.8).

Problem 3. Let $T$ be a power-bounded operator in $H$ such that $T$ commutes with a compact operator $K$ with $\operatorname{ker}(K)=\{0\}$. Is $T$ similar to a contraction?

By considering $T^{*}$ instead of $T$, one can replace the condition $\operatorname{ker}(K)=\{0\}$ by $\overline{\operatorname{ran}(K)}=H$. Then the semigroup $\left\{T^{n}: n \in \mathbb{Z}_{+}\right\}$is almost periodic, so Problem 3 is reduced to the particular case when $T$ is strongly stable. Note also that if $T$ is power-bounded (on a Banach space $X$ ) and commutes with a compact operator with 
$\overline{\operatorname{ran}(K)}=X$, and if $P \sigma(T) \cap \mathbb{T}$ is empty, then $T$ is strongly stable, since it is almost periodic with zero boundary subspace [46]. Therefore, the stability problem is easily solved (by de Leeuw-Glicksberg Decomposition) in the class of operators commuting with a compact operator, a fact which says that commuting with a compact operator is a strong property.

It is known that if $T$ is compact and power-bounded, then $T$ is similar to a contraction (see [35]). If $r(T)<1$, where $r(T)$ is the spectral radius of $T$, then $T$ also is similar to a contraction (Rota's Theorem, see e.g. [22]). A synthesis of these two facts was given in [47] where it was shown that if a power-bounded operator $T$ is a sum of a compact operator and an operator with spectral radius $<1$, then $T$ is similar to a contraction. On the other hand, the analog of Rota's Theorem does not hold for $C_{0}$-semigroups: Chernoff [11] has given an example of exponentially stable $C_{0}$-semigroup which is not similar to a contraction semigroup. Moreover, McIntosh and Yagi [32] and Baillon and Clément [4] contain implicitly examples of exponentially stable analytic semigroups which are not similar to contraction semigroups (since they do not have $H^{\infty}$-functional calculus) ( ${ }^{6}$ ). This is another evidence of the well known fact that the theory of $C_{0}$-semigroups is not "isomorphic" to the theory of single operators. The generators in Chernoff's, BaillonClément's and McIntosh-Yagi's examples must be unbounded: it is also shown in [47] that if $T(t)$ is a bounded $C_{0}$-semigroup such that the generator $A$ is bounded, and if $T\left(t_{0}\right)$ (for some $t_{0}>0$ ) is a sum of a compact operator and an operator with spectral radius less than 1 , then $T(t)$ is similar to a contraction semigroup. It remains an open question, however, whether every bounded compact or eventually compact $C_{0}$-semigroup (with unbounded generator) is similar to a contraction semigroup. In particular, is every nilponent $C_{0}$-semigroup similar to a contraction semigroup?

Now let $S$ be a suitable subsemigroup of a locally compact abelian group $G$ and $T$ be a representation of $S$ on $L(X)$. Theorem 4.5 suggests the following generalization of the Sz.-Nagy-Foiaş Theorem.

Problem 4. Assume that $\{T(s): s \in S\}$ is a c.n.u. contraction representation, and $m\left(\operatorname{Sp}(T) \cap S_{\mathrm{u}}^{*}\right)=0$, where $m$ is the Haar measure on $G^{*}$. Is $T$ strongly stable?

The simplest unsolved partial case of Problem 4 is the case of several commuting contractions, which can be formulated as follows: Let $\left\{T_{1}, \ldots, T_{m}\right\}$ be a finite family of commuting contractions on a Hilbert space $H$ which is completely nonunitary (i.e. there is no common reducing subspace $K \neq\{0\}$ on which $T_{i}$ are unitary, $\left.\forall i\right)$. Let $\sigma\left(T_{1}, \ldots, T_{m}\right)$ be the joint spectrum of $T_{1}, \ldots, T_{m}$. Assume that $m\left(\sigma\left(T_{1}, \ldots, T_{m}\right) \cap \mathbb{T}^{m}\right)=$ 0 , where $m$ is the normalized Lebesgue measure on $\mathbb{T}^{m}$. Is $\left\{T_{1}, \ldots, T_{m}\right\}$ jointly stable, i.e. $\lim _{k \rightarrow \infty}\left\|T_{1}^{k} \ldots T_{m}^{k} x\right\|=0, \forall x \in H$ ?

Problem 5. Let $\{T(s): s \in S\}$ be a bounded representation of $S$ on $H$ such that $T\left(s_{0}\right)$ is compact and $\operatorname{ker}\left(T\left(s_{0}\right)\right)=\{0\}$ for some $s_{0} \in S$. Is $T$ similar to a contraction representation?

$\left({ }^{6}\right)$ The papers [4] and [32] and their connection with the similarity problem were brought to the author's attention by R. deLaubenfels. 
Acknowledgements. I am very thankful to Professors R. deLaubenfels and Wang Shengwang for many helpful discussions on the topics of this article.

\section{References}

[1] W. Arendt and C. J. K. Batty, Tauberian theorems and stability of one-parameter semigroups, Trans. Amer. Math. Soc. 306 (1988), 837-852.

[2] R. Arens, Inverse-producing extensions of normed algebras, Trans. Amer. Math. Soc. 88 (1958) 536-548.

[3] W. B. Arveson, On groups of automorphisms of operator algebras, J. Funct. Anal. 15 (1974), 217-243.

[4] J. B. Baillon and P. Clément, Examples of unbounded imaginary powers of operators, ibid. 100 (1991), 419-434.

[5] W. Bartoszek, Asymptotic periodicity of the iterates of positive contractions on Banach lattices, Studia Math. 91 (1988), 179-188.

[6] C. J. K. Batty, Asymptotic behaviour of semigroups of operators in: Functional Analysis and Operator Theory, Banach Center Publ. 30, Inst. Math., Polish Acad. Sci., Warszawa, 1994, 35-52.

[7] C. J. K. Batty, Z. Brzeźniak and D. Greenfield, A quantitative asymptotic theorem for contraction semigroups with countable unitary spectrum, Studia Math. 121 (1996), $167-183$.

[8] C. J. K. Batty and Vũ Quôc Phóng, Stability of individual elements under oneparameter semigroups, Trans. Amer. Math. Soc. 322 (1990), 805-818.

[9] —, - Stability of strongly continuous representations of abelian semigroups, Math. Z. 209 (1992), 75-88.

[10] B. Beauzamy, Introduction to Operator Theory and Invariant Subspaces, NorthHollland, Amsterdam, 1988.

[11] P. R. Chernoff, Two counterexamples in semigroup theory on Hilbert space, Proc. Amer. Math. Soc. 56 (1976), 253-255.

[12] I. Colojoară and C. Foiaş, Theory of Generalized Spectral Operators, Gordon \& Breach, New York, 1968.

[13] R. deLaubenfels, Existence Families, Functional Calculi and Evolution Equations, Lecture Notes in Math. 1570, Springer, Berlin, 1994.

[14] R. deLaubenfels and Vũ Quôc Phóng, Stability and almost periodicity of solutions of ill-posed abstract Cauchy problems, Proc. Amer. Math. Soc., to appear.

[15] - - - The discrete Hille-Yosida space, stability of individual orbits, and invariant subspaces, preprint.

[16] R. G. Douglas, On extending commutative semigroups of isometries, Bull. London Math. Soc. 1 (1969), 157-159.

[17] I. Erdelyi and S. W. Wang, A Local Spectral Theory for Closed Operators, London Math. Soc. Lecture Note Ser., Cambridge Univ. Press, Cambridge, 1985.

[18] J. Esterle, E. Strouse et F. Zouakia, Stabilité asymptotique de certains semigroupes d'opérateurs, J. Operator Theory 28 (1992), 203-227.

[19] S. R. Foguel, A counterexample to a problem of Sz.-Nagy, Proc. Amer. Math. Soc. 15 (1964), 788-790.

[20] I. Gelfand, Zur Theorie der Charaktere der abelschen topologischen Gruppen, Mat. Sb. 9 (51) (1941), 49-50. 
[21] P. R. Halmos, On Foguel's answer to Nagy's question, Proc. Amer. Math. Soc. 15 (1964), 791-793.

[22] - - A Hilbert Space Problem Book, Springer, Berlin, 1982.

[23] E. Hille and R. S. Phillips, Functional Analysis and Semi-Groups, Amer. Math. Soc., Providence, R.I., 1957.

[24] Y. Katznelson and L. Tzafriri, On power bounded operators, J. Funct. Anal. 68 (1986), 313-328.

[25] L. Kérchy, Unitary asymptotes of Hilbert space operators, in: Functional Analysis and Operator Theory, Banach Center Publ. 30, Inst. Math., Polish Acad. Sci., Warszawa, 1994, 191-201.

[26] U. Krengel, Ergodic Theorems, de Gruyter, Berlin, 1985.

[27] A. Lasota, T. Y. Li and J. A. Yorke, Asymptotic periodicity of the iterates of Markov operators, Trans. Amer. Math. Soc. 286 (1984), 751-764.

[28] Yu. I. Lyubich, On the spectrum of a representation of an abelian topological group, Dokl. Akad. Nauk SSSR 12 (1971), 1482-1486 (in Russian).

[29] Yu. I. Lyubich, V. I. Matsaev and G. M. Fel'dman, Representations with separable spectrum, Funct. Anal. Appl. 7 (1973), 129-136.

[30] Yu. I. Lyubich, Introduction to the Theory of Banach Representations of Groups, Birkhäuser, Basel, 1988.

[31] Yu. I. Lyubich and Vũ Quôc Phóng, Asymptotic stability of linear differential equations in Banach spaces, Studia Math. 88 (1988), 37-42.

[32] A. McIntosh and A. Yagi, Operators of type $\omega$ without a bounded $H_{\infty}$-functional calculus, in: Miniconference on Operators in Analysis, Proc. Centre Math. Anal., ANU, Canberra (1989) 24, 159-172.

[33] M. Miklavčič, Asymptotic periodicity of the iterates of positivity preserving operators, Trans. Amer. Math. Soc. 307 (1988), 469-479.

[34] B. Sz.-Nagy and C. Foiaş, Harmonic Analysis of Operators on Hilbert Space, NorthHolland, Amsterdam, 1970.

[35] B. Sz.-Nagy, Completely continuous operators with uniformly bounded iterates, Magyar Tud. Akad. Mat. Kutató Int. Közl. 4 (1959), 89-93.

[36] E. W. Packel, A semi-group analogue of Foguel's counterexample, Proc. Amer. Math. Soc. 21 (1969), 240-244.

[37] M. Putinar, Hyponormal operators are subscalar, J. Operator Theory 12 (1984), 385395.

[38] C. R. Putnam, Hyponormal contractions and strong power convergence, Pacific J. Math. 57 (1975), 531-538.

[39] G. M. Sklyar and V. Ya. Shirman, On the asymptotic stability of linear differential equations in a Banach space, Teor. Funktsiǔ Funktsional. Anal. i Prilozhen. 37 (1982), 127-132.

[40] Vũ Quôc Phóng and Yu I. Lyubich, A spectral criterion for asymptotic almost periodicity of uniformly continuous representations of abelian semigroups, J. Soviet Math. 51 (1990), 1263-1266. Originally published in Teor. Funktsiu Funktsional. Anal. i Prilozhen. 50 (1988), 38-43 (in Russian).

[41] - - - A spectral criterion for almost periodicity of one-parameter semigroups, J. Soviet Math. 48 (1990), 644-647. Originally published in Teor. Funktsiü Funktsional. Anal. i Prilozhen. 47 (1987), 36-41 (in Russian). 
[42] Vũ Quôc Phóng, Theorems of Katznelson-Tzafriri type for semigroups of operators, J. Funct. Anal. 103 (1992), 74-84.

[43] - , A short proof of the Y. Katznelson's and L. Tzafriri's theorem, Proc. Amer. Math. Soc. 115 (1992), 1023-1024.

[44] - On the spectrum, complete trajectories and asymptotic stability of linear semidynamical systems, J. Differential Equations 105 (1993), 30-45.

[45] - Asymptotic almost periodicity and compactifying representations of semigroups, Ukrain. Math. J. 38 (1986), 576-579.

[46] - Stability of $C_{0}$-semigroups commuting with a compact operator, Proc. Amer. Math. Soc., to appear.

[47] Vũ Quôc Phóng and F. Y. Yao, On similarity to contraction semigroups on Hilbert space, Semigroup Forum, to appear.

[48] J. Wermer, Banach Algebras and Several Complex Variables, Springer, New York, 1976.

[49] W. Żelazko, On a certain class of non-removable ideals in Banach algebras, Studia Math. 44 (1972), 87-92.

[50] J. Zemánek, On the Gelfand-Hille theorems, in: Functional Analysis and Operator Theory, Banach Center Publ. 30, Inst. Math., Polish Acad. Sci., Warszawa, 1994, 369-385. 